\title{
An examination of the reciprocal and concurrent relations between behavioral and cardiac indicators of acute pain in toddlerhood
}

\author{
Jordana A. Waxmana , Miranda G. DiLorenzo ${ }^{a}$, Rebecca R. Pillai Riddell ${ }^{a, b, c, \star}$, David B. Flora ${ }^{a}$, Louis A. Schmidt ${ }^{d}$,
} Hartley Garfield ${ }^{\mathrm{b}, \mathrm{c}}$, Dan Flanders ${ }^{\mathrm{c}}$, Eitan Weinberg ${ }^{\mathrm{c}}$, Deena Savlov ${ }^{\mathrm{c}}$

\begin{abstract}
The aim of this study was to examine the concurrent and predictive relations between healthy toddlers' pain behavior and cardiac indicators (ie, heart rate [HR] and respiratory sinus arrhythmia [RSA]) during routine vaccinations. Caregiver-infant dyads were part of a longitudinal cohort observed during their 12- and 18-month vaccinations. Behavioral and cardiac data were simultaneously collected for 1-minute preneedle and 3-minutes postneedle. Videotapes were coded for pain behaviors (FLACC; Merkel et al., 1996), and cardiac data were analyzed (HR, RSA) during sequential 30-second epochs. Four separate cross-lagged path models were estimated using data from the 12- $(n=147)$ and 18-month $(n=122)$ vaccinations. Across 12 - and 18-month vaccinations, predictive within-measure relations were consistent for FLACC, HR, and RSA, reflecting good stability of these pain indicators. Behavioral indicators predicted subsequent HR and RSA within the immediate postneedle period. Both baseline behavior and HR/RSA predicted future pain scores. Concurrent residual relations between behavioral and cardiac indicators were inconsistent across time and indicators. Results suggest that behavioral and cardiac indicators reflect unique aspects of the nociceptive response. As such, multimodal assessment tools should be used and contextualized by child age, cardiac indicator, baseline behavior/physiology, and pain phase.
\end{abstract}

Keywords: Acute pain, Behavior, Child development, Heart rate, Heart rate variability, Toddlerhood

\section{$A Q: 4$ 1. Introduction}

It is well established that very young children can experience pain, with pain transmission pathways in the brain being fully developed by 22 to 24 weeks of gestation. ${ }^{49}$ Exposure to repeated painful experiences in infancy has been reliably linked to altered brain development and pain intensity as well as poor early neurodevelopment and quality of cognitive and motor development. ${ }^{48,54}$ A challenge with pain management in young children is that despite knowing that early painful experiences impact a child's physical and neuropsychological development, there is currently no gold-standard pain indicator because reliable selfreport does not occur until approximately 6 to 7 years of age. ${ }^{56}$

When infants and young children are hospitalized, in the absence of self-report measures, current clinical pain scoring systems rely on multiple indicators that incorporate behavioral (eg, facial expression and body movements) and physiological responses (eg, heart rate

AQ:5 [HR] and oximetry). ${ }^{45}$ Although some indicators have been validated in clinical samples, ${ }^{10,20,22,25,38}$ scores derived from these indicators

Sponsorships or competing interests that may be relevant to content are disclosed $\mathrm{AQ}: 2$ at the end of this article.

a York University, Toronto, ON, Canada, ${ }^{b}$ The Hospital for Sick Children, Toronto, ON, Canada, ${ }^{\circ}$ University of Toronto, Toronto, ON, Canada, ${ }^{d}$ McMaster University,

AQ:1 Hamilton, ON, Canada

${ }^{*}$ Corresponding author. Address: Department of Psychology, York University, 4700 Keele St, OUCH Laboratory, 2004/6 Sherman Health Sciences Building, Toronto, ON M3J 1P3, Canada. Tel.: 416-736-2100 x 20177; fax: 416-736-5772. E-mail address: rpr@yorku.ca (R.R. Pillai Riddell).

PAIN 00 (2020) 1-15

(C) 2020 International Association for the Study of Pain

http://dx.doi.org/10.1097/j.pain.0000000000001840 have not consistently converged with pain-specific cortical activity. ${ }^{50}$ This discrepancy reflects the possibility that behavioral and physiological measures of pain-related distress each represent important, unique information about the nociceptive response in infancy and toddlerhood. ${ }^{11}$

In most empirical work focusing on underlying physiological components of distress, maturation of the autonomic nervous system is highlighted as fundamental for emotion regulation. ${ }^{46}$ Indeed, pain scales that include both behavioral and cardiac indicators are pervasive in the hospital setting. However, little research has examined how behavioral and cardiac responses to acutely painful procedures converge after the first 4 months of life. ${ }^{58}$ Results from the little available research are equivocal with studies finding small-to-moderate positive correlations ${ }^{34,44}$ or describing nonsignificant or divergent responses after acute pain. ${ }^{16,45}$ Longitudinal research examining behavioral pain-related distress responses in infancy found differences in behavioral pain-related distress by 12 months of age, which were posited to be due to traitlike differences in negative affect regulation, distress, or pain responding. ${ }^{41}$ The sensitivity of cardiac indicators in response to acute pain in later infancy and toddlerhood is unknown. This association is the focus of the current analyses.

Roué et al. ${ }^{45}$ have called for more research on improving measurement of behavioral and physiological responses to best encompass an infant's pain-related distress. However, the convergence of different indicators first needs to be established in healthy samples to provide a knowledge base. ${ }^{45}$ The current study examines the predictive and concurrent within- and betweenmeasure and contextual (ie, baseline responses and time since last feeding and nap) relations between toddlers' expressed pain behaviors and cardiac responses (ie, HR and RSA) during 12- and 
18-month vaccinations. Predictive and concurrent relations were examined because recent research has found that individual differences in expressed emotion are associated with differences in cardiac reactivity and recovery across distressing events (ie, anger induction) in toddlerhood. ${ }^{26}$ The 12- and 18-month ages were investigated separately because cognitive and physical development has been characterized by more differentiated behavioral and physiological responses at 18 months than at 12 months. ${ }^{1,31}$ We hypothesized that preceding expressed pain-related responses (behaviors or cardiac responses) would predict subsequent expressed pain-related responses (behaviors or cardiac responses) within an indicator (eg, behavior predicting behavior), given that previous research in typically developing infants has found that earlier infant pain behavior is a strong predictor of subsequent infant pain behavior within the immunization context. ${ }^{9}$ Small associations have been found between expressed emotion and cardiac indicators of distress (ie, fear and frustration) measured concurrently (ie, during the same visit) in toddlerhood. ${ }^{57}$ As such, we hypothesized that expressed pain-related responses (behaviors or cardiac responses) would predict subsequent expressed pain-related responses between indicators (eg, behavior predicting HR). Because HR and RSA reflect largely sympathetic and parasympathetic functioning, respectively, ${ }^{4,6}$ we hypothesized positive relations between behavior and $\mathrm{HR}$ and negative relations between behavior and RSA.

\section{Method}

\subsection{Participants}

Ethical approval was obtained through the research ethics review board at the participating university. After agreeing to speak to a researcher about the study, caregivers were approached by a research assistant who explained the study and then asked them to sign informed consent forms.

The data are part of an ongoing longitudinal study in which caregiver-toddler dyads were recruited from 2 pediatric clinics in the greater Toronto area and observed with a cohort-sequential design during vaccinations over the second year of life (12, 18, and 24 months). Toddlers were recruited at 12 or 18 months of age. Of the 374 families approached for recruitment at 12 or 18 months, 41 were ineligible based on exclusion criteria (ie, child was hospitalized in a neonatal intensive care unit, was more than 3 weeks premature, suspected of a developmental delay, had a known heart condition, or the caregiver was not fluent in English). In total, 158 and 122 caregiver-toddler dyads were successfully recruited at the 12- and 18-month vaccinations, respectively. A total of 72 participants were observed at both the 12- and 18-month vaccinations. Table 1 lists demographic characteristics of the participants included. Overall, participants were healthy, from middle-class families, and had well-educated caregivers.

Participants had diverse cultural backgrounds. Many of the primary caregivers were born in Canada (59\%), yet a substantial percentage of caregivers were born outside of Canada (Asia [23\%], Europe [9\%], South America [6\%], Australia [2\%], or United States [1\%]). In addition, their rating of acculturation suggested an integrated cultural background, with strong identification with both their heritage culture (a culture that influenced generations of their family) and mainstream Canadian culture (the culture in which they currently live).

\subsection{Procedure}

Caregivers filled out a short demographic questionnaire before each vaccination appointment. During each vaccination, caregivertoddler dyads were simultaneously videotaped and connected to
Table 1

\section{Demographic information.}

\begin{tabular}{|c|c|c|}
\hline & \multicolumn{2}{|c|}{ Frequency (\%) } \\
\hline & $\begin{array}{l}12 \mathrm{mo} \\
(\mathrm{n}=158)\end{array}$ & $\begin{array}{l}18 \mathrm{mo} \\
(\mathrm{n}=122)\end{array}$ \\
\hline \multicolumn{3}{|l|}{ Sex of infant } \\
\hline Male & $81(54.4)$ & $68(58.1)$ \\
\hline Female & $68(45.6)$ & 49 (41.9) \\
\hline \multicolumn{3}{|l|}{ Relationship to infant } \\
\hline Mother & 127 & 98 \\
\hline Father & 12 & 17 \\
\hline Other & 1 & 1 \\
\hline \multicolumn{3}{|l|}{ Education } \\
\hline Graduate school/professional training & 72 & 55 \\
\hline University graduate (4 y) & 42 & 39 \\
\hline Partial university (at least 1 y) & 4 & 3 \\
\hline Trade school/community college & 15 & 11 \\
\hline High school graduate & 2 & 1 \\
\hline Age & $35.91(5.19)$ & $36.63(6.19)$ \\
\hline \multicolumn{3}{|l|}{ Acculturation status } \\
\hline Way of life reflects heritage culture & $6.63(2.79)$ & $5.66(2.43)$ \\
\hline $\begin{array}{l}\text { Way of life reflects mainstream North } \\
\text { American/Canadian culture }\end{array}$ & $7.62(2.28)$ & $7.75(1.66)$ \\
\hline
\end{tabular}

equipment to measure their HR before and after the child's vaccinations. Based on methodology from a previous longitudinal infant cohort followed during their well-baby visits, ${ }^{41}$ at both the 12 and 18-month vaccinations, toddlers were observed 1 minute before, immediately after the final needle, 1 minute after the final needle, and 2 minutes after the final needle. Noldus and MindWare technologies were used to synchronize acquisition and analysis of the physiological data and video recordings. The dyads were observed with minimal interference from the research team aside from videotaping and the cardiac monitoring procedures. At both the 12- and 18-month vaccinations, caregivers were given a sheet outlining evidence-based pain management strategies (3 Ps of Helping your Child during Vaccinations A Parent's Guide: Children over 1 year old ${ }^{52}$ ).

\subsection{Measures}

\subsubsection{Caregiver demographic information}

Caregivers were asked to complete a short demographic questionnaire that asked for caregiver age, relation to the child, self-reported heritage culture, and child age and sex. Caregivers were also asked to report important infant factors that are known to impact physiological indicators, ${ }^{36,52}$ such as time since last feeding and since last nap (parent report in minutes).

\subsubsection{Pain behaviors}

The Face, Legs, Activity, Cry, Consolability coding system (FLACC ${ }^{32}$ ) was used to assess the degree of behavioral pain across the vaccination appointments. The degree of behavioral pain was measured with 5 types of pain behaviors (face, legs, activity, cry, and consolability) during 7 different epochs (60 to 1 second before the first needle [FLACCB]; 0-29 seconds immediately after the last needle [FLACCO]; 30-59 seconds after the last needle [FLACC1]; 60-89 seconds after the last needle [FLACC2]; 90-119 seconds after the last needle [FLACC3]; 120-149 
seconds after the last needle [FLACC4]; and finally, 150-179 seconds after the last needle [FLACC5]). Each behavior was scored with a 0 to 2 scale (eg, on the Face scale, no expression or smile is scored 0 , occasional observations of certain facial expressions [ie, grimace, frown], or the child being withdrawn is scored 1, and constant frown, clenched jaw, or quivering chin is scored 2), resulting in possible total scores between 0 and 10 for each epoch. There were no significant differences between the two 30-second baseline epochs, and so they were averaged to provide a more robust baseline indicator. Moderate to high concurrent validity as well as item-total and interrater reliability has been demonstrated for FLACC scores in the acute pain context. ${ }^{33}$ To ensure high reliability, coders were trained by a primary FLACC coder. A total of $20 \%$ of the sample was reliability-coded throughout the coding process, with unreliable codes (ie, intraclass correlation for a given epoch below 0.8 ) being consensus coded with the primary and reliability coders present. This is a rare occurrence because interrater reliability between the coders was high (intraclass correlations between 0.9 and 0.93). The coders were blinded to the study hypotheses.

\subsubsection{Cardiac indicators: heart rate and respiratory sinus arrhythmia}

Cardiac data were collected continuously using MindWare ambulatory monitors (MW 1000A) at a sampling rate of $500 \mathrm{~Hz}$. Three adhesive electrodes collected electrocardiography (ECG), with one electrode placed above the right shoulder blade, one electrode placed on the bottom-most left rib, and a ground electrode placed on the bottom-most right rib. Using MindWare BioLab 3.3, ECG signals were continuously acquired. Electrocardiography data were edited in MindWare HRV 3.1.5, with HR computed through identification of R-waves, and spectral analysis of the ECG data being used to compute respiratory sinus arrhythmia (RSA). ${ }^{4}$ We used a frequency band of 0.24 to $1.04 \mathrm{~Hz}$ to quantify RSA within the range of spontaneous respiration in young children. ${ }^{22}$ To ensure high reliability, coders were trained by an experienced primary coder. A total of $20 \%$ of the sample was reliability-coded throughout the coding process, with unreliable codes (ie, intraclass correlation for a given epoch below 0.9) being recoded after consultation with the primary coder. The coders were blinded to the study hypotheses, and interrater reliability between the coders was high (intraclass correlations between 0.95 and 0.99 ).

Trained coders identified any misidentified R-waves from the raw physiological data. Editing issues (eg, cutting segments of data and identifying R-waves on data with artifact) were addressed and corrected in consultation with the experienced primary coder. In the case of artifact, the decision to include the data was made on an epoch-by-epoch basis in consultation with the primary coder. The primary reason for excluding an epoch of HR/RSA data was serial missing R-waves (where a "midbeat" could not be estimated). In all cases, the key decision rule was whether edited epochs were consistent with the individual's other

[T2] portions of data. Table 2 provides a breakdown of the reasons toddlers' HR/RSA data were not used, that were not a result of editing challenges. The amount of artifact editing did not exceed $5 \%$ and did not systematically relate to any of the study measures.

Cardiac values (ie, HR and RSA) were calculated during 7 different epochs (60 to 0 seconds before the first needle [HRB, RSAB]; 0-30 seconds immediately after the last needle [HRO, RSA0]; 30-60 seconds after the last needle [HR1, RSA1]; 60-90 seconds after the last needle [HR2, RSA2]; 90-120 seconds after the last needle [HR3, RSA3]; 120-150 seconds after the last
Table 2

Reasons for cardiac data not being usable.

\begin{tabular}{lll}
\hline & Frequency & \\
\cline { 2 - 3 } & $\mathbf{1 2} \mathbf{~ m o}$ & $\mathbf{1 8 ~ \mathbf { ~ o }}$ \\
\hline Complete & 129 & 101 \\
\hline Device malfunction & 19 & 15 \\
\hline Timing & 5 & 4 \\
\hline No needle & 2 & 2 \\
\hline Blocked & 1 & 0 \\
\hline Electrode removed & 1 & 4 \\
\hline Declined stickers & 0 & 1 \\
\hline Lost to follow-up & 0 & 23 \\
\hline Refused & 0 & 14 \\
\hline
\end{tabular}

needle [HR4, RSA4]; and 150-180 seconds after the last needle [HR5, RSA5]). Because there were no significant differences between two 30-second baseline epochs, they were collapsed to provide a more robust baseline indicator. Inclusion of a 60second baseline epoch is consistent with the Task Force standards of measurement, physiological interpretation, and clinical use of HRV data, ${ }^{35,53}$ which states that approximately 1 minute of data are needed to assess the high-frequency components of HRV (ie, RSA). Heart rate indicators significantly differed across 30-second postneedle epochs, and thus no postneedle epochs were combined.

\subsection{Analysis plan}

To examine reciprocal influences on toddler's expressed pain behaviors and cardiac responses (ie, HR, RSA) in the 12- and 18month vaccination contexts, 4 autoregressive cross-lagged path models [see Ref. 28 for review of the statistical approach] (Figs. 1-4) were estimated using structural equation modeling software using a robust full-information maximum likelihood estimator to incorporate incomplete cases and account for the degree of nonnormality in the data. These models were specified so that for both toddlers' behavioral pain and cardiac responses, 3 types of relations were examined simultaneously: (1) Predictive Within-Measure: the prediction of behavioral pain response (or cardiac response) from the behavioral pain response (or cardiac response) that directly preceded it (eg, RSA immediately postneedle [RSAO] predicting RSA 30 seconds after the needle [RSA1]); (2) Predictive Between-Measure: the prediction of a behavioral pain response (or cardiac response) from the cardiac response (or behavioral pain response) that directly preceded it (eg, HR immediately postneedle [HRO] predicting behavioral pain 30 seconds after the needle [FLACC1]); (3) Concurrent (Residual) Between-Measure: the concurrent residual relations between behavioral pain response and cardiac responses at baseline and each of the 6 postneedle epochs, after controlling for their predictors in the model (eg, behavioral pain response immediately after the last needle [FLACCO] with RSA immediately after the last needle [RSA0], after accounting for baseline variables).

A final analysis examined contextual factors that may impact the relation between behavioral pain and cardiac responses. Specifically, baseline responses (ie, baseline behavioral pain responses, baseline HR, and baseline RSA) were included in the initial autoregressive cross-lagged models as covariates, given that the Law of Initial Value 


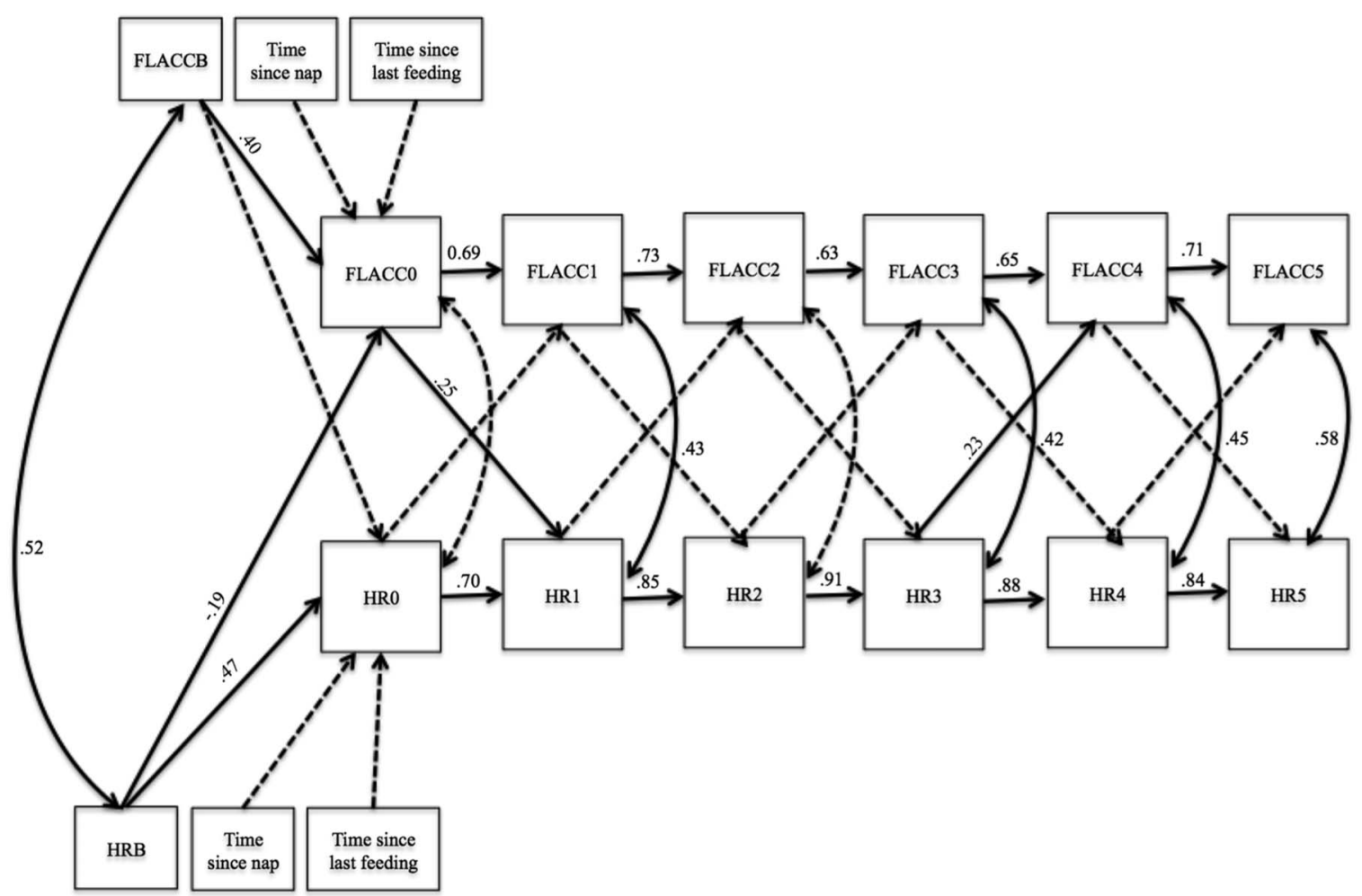

Figure 1. Autoregressive cross-lagged path model: relations between behavioural pain scores and heart rate during 12-month vaccination. Solid paths are significant with $P<0.01$. Nonsignificant paths are dashed.

asserts that the size of a psychophysiological response depends on the initial baseline level of the measure. ${ }^{5}$ In addition, level of arousal has recently been shown to be a determinant of pain-related brain activity. ${ }^{24}$ Calculation of baseline responses was outlined in sections 2.3.2 and 2.3.3.

Time since last feeding and time since last nap (in minutes)

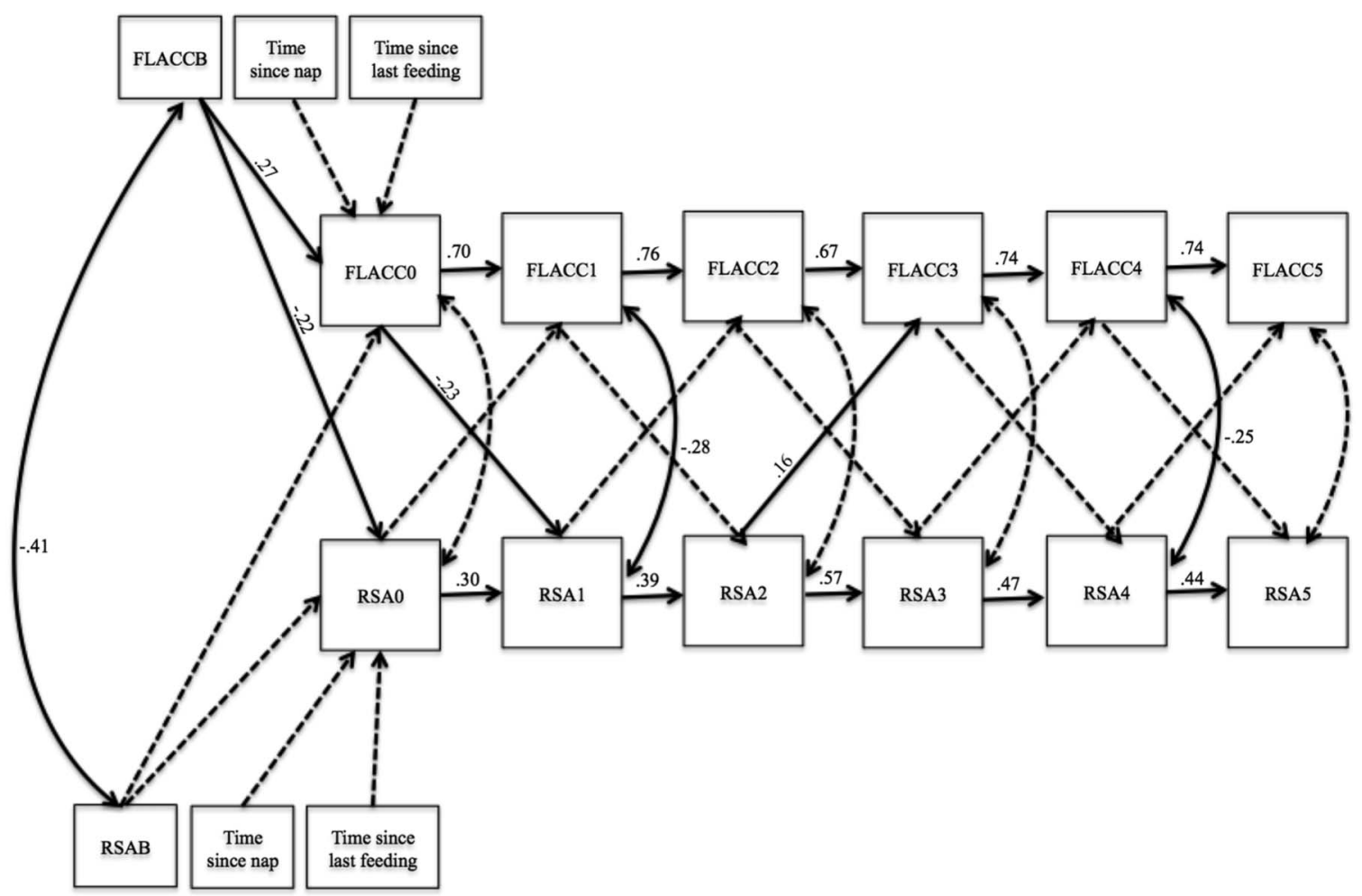

Figure 2. Autoregressive cross-lagged path model: relations between behavioural pain scores and respiratory sinus arrhythmia during 12 -month vaccination. Solid paths are significant with $P<0.01$. Nonsignificant paths are dashed. 


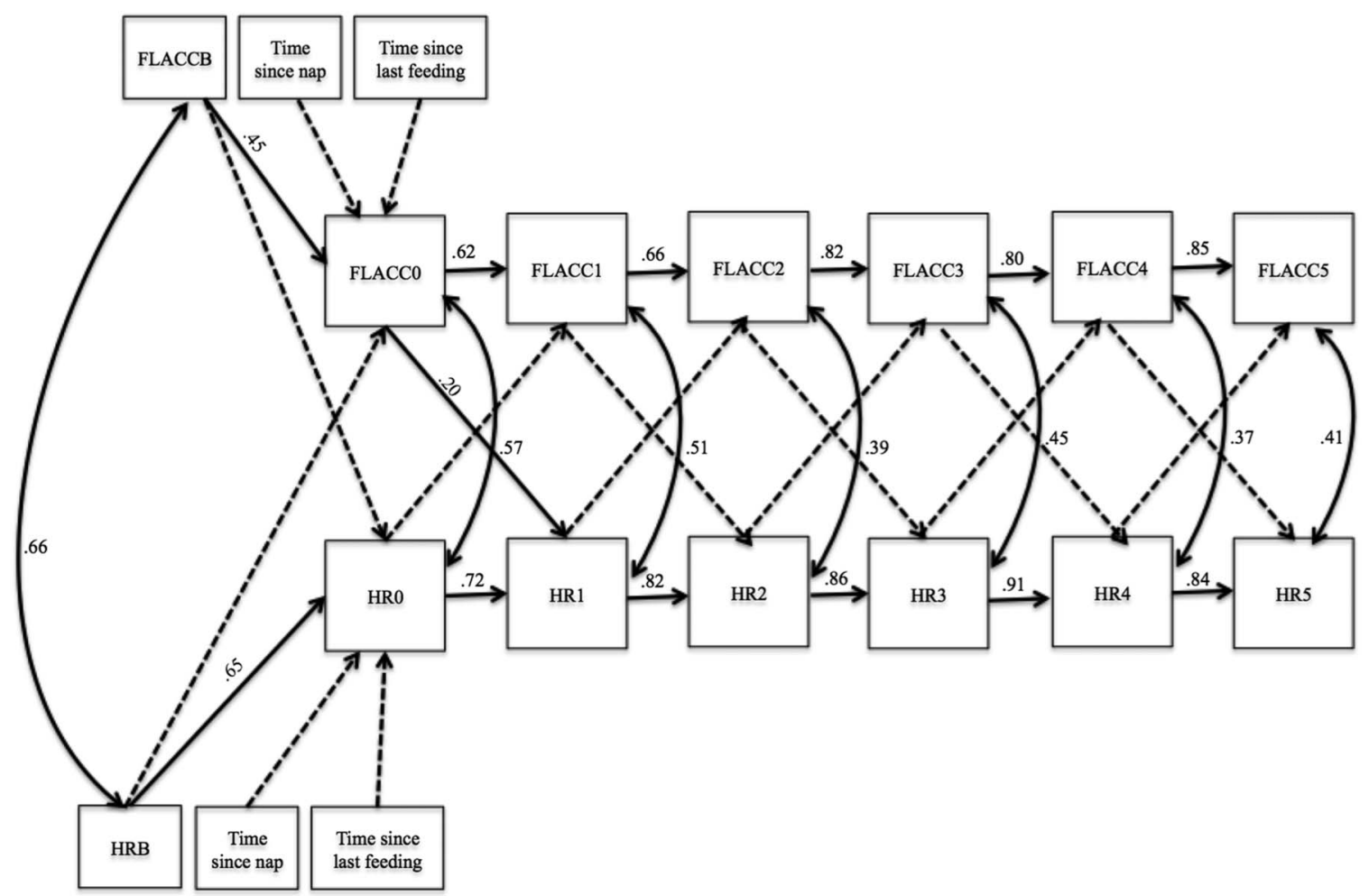

Figure 3. Autoregressive cross-lagged path model: relations between behavioural pain scores and heart rate during 18-month vaccination. Solid paths are significant with $P<0.05$. Nonsignificant paths are dashed.

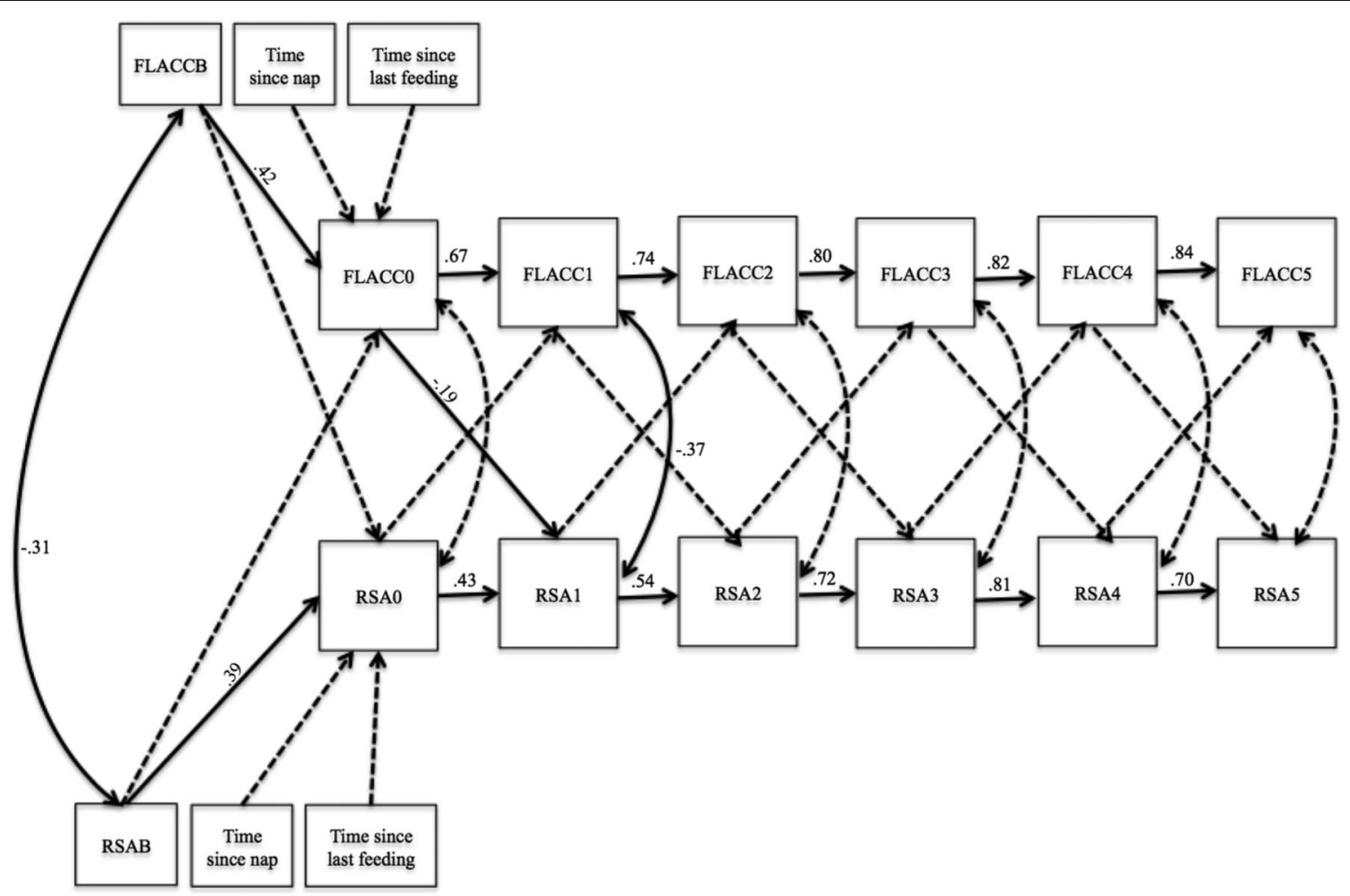

Figure 4. Autoregressive cross-lagged path model: relations between behavioural pain scores and respiratory sinus arrhythmia during 18-month vaccination. Solid paths are significant with $P<0.01$. Nonsignificant paths are dashed. 
were also included in the initial model as covariates because these factors are known to impact infant physiology. ${ }^{36,58}$

\section{Results}

[T3 - T4] Tables 3 and 4 present the mean values and SDs of all variables [T5 - T8] in the 12- and 18-month models, and Tables 5-8 present the correlations among all variables in the 12- and 18-month models. Because full-information maximum likelihood was used, the models were fitted to 147 and 122 participants at 12 and 18 months, respectively.

\subsection{The relations between toddlers' behavioral and cardiac responses during 12-month vaccinations}

\subsubsection{Relations between pain behaviors and heart rate}

The autoregressive cross-lagged path model in Figure 1 fits the data adequately $(\mathrm{CFI}=0.94$; RMSEA $=0.08)$. Standardized estimates of significant paths are reported in Figure 1, and all

[T9] standardized and unstandardized estimates are reported in Table 9.

\subsubsection{Predictive within-measure}

Across the vaccination period at 12 months, each postneedle behavioral response significantly positively predicted the subsequent behavioral response (standardized $\mathrm{Bs}=0.63-0.72$ ),

\section{Table 3}

Mean values and SDs of variables used for 12-month models.

\begin{tabular}{|c|c|c|c|}
\hline & Mean & SD & Scale range \\
\hline Time since last feeding (min) & 103.97 & 74.94 & $0-420$ \\
\hline Time since last nap (min) & 110.40 & 76.71 & $0-390$ \\
\hline FLACCB & 1.68 & 1.83 & $0-8$ \\
\hline FLACCO & 7.30 & 1.55 & $1-10$ \\
\hline FLACC1 & 6.28 & 2.5 & $0-10$ \\
\hline FLACC2 & 5.21 & 2.86 & $0-10$ \\
\hline FLACC3 & 4.2 & 2.85 & $0-9.5$ \\
\hline FLACC4 & 3.39 & 2.84 & $0-9.5$ \\
\hline FLACC5 & 3.03 & 2.85 & $0-9$ \\
\hline HRB & 129.92 & 13.94 & 81.17-180.48 \\
\hline HRO & 150.82 & 21.40 & $84.54-207.41$ \\
\hline HR1 & 154.59 & 22.90 & 77.45-191.58 \\
\hline HR2 & 146.06 & 20.90 & 85.89-195.91 \\
\hline HR3 & 140.36 & 18.66 & 84.12-193.89 \\
\hline HR4 & 136.38 & 17.53 & 80.03-183.94 \\
\hline HR5 & 134.07 & 17.08 & 77.54-191.58 \\
\hline RSAB & 3.89 & 1.18 & $1.12-7.85$ \\
\hline RSAO & 4.23 & 2.23 & $0-9.69$ \\
\hline RSA1 & 2.99 & 1.54 & $0-6.71$ \\
\hline RSA2 & 3.43 & 1.32 & $0.11-6.81$ \\
\hline RSA3 & 3.87 & 1.23 & $0.64-7.32$ \\
\hline RSA4 & 3.81 & 1.13 & $0.88-6.36$ \\
\hline RSA5 & 3.93 & 1.31 & $1.04-8.66$ \\
\hline
\end{tabular}

FLACC, Face, Legs, Activity, Cry, Consolability scale; HR, heart rate (beats per minute); RSA, respiratory sinus arrhythmia.
Table 4

\section{Mean values and SDs of variables observed at 18-month} models.

\begin{tabular}{|c|c|c|c|}
\hline & Mean & SD & Scale range \\
\hline Time since last feeding (min) & 100.12 & 61.16 & $0-300$ \\
\hline Time since last nap (min) & 148.83 & 85.70 & $0-420$ \\
\hline FLACCB & 2.81 & 2.71 & $0-9.75$ \\
\hline FLACCO & 6.46 & 2.11 & $0-9$ \\
\hline FLACC1 & 5.10 & 2.91 & $0-9.38$ \\
\hline FLACC2 & 4.37 & 2.90 & $0-9$ \\
\hline FLACC3 & 3.81 & 2.84 & $0-9.17$ \\
\hline FLACC4 & 3.38 & 2.82 & $0-9.5$ \\
\hline FLACC5 & 3.34 & 2.02 & $0-10$ \\
\hline HRB & 132.01 & 17.5 & 102.78-186.02 \\
\hline HRO & 147.80 & 23.27 & $98.66-198.20$ \\
\hline HR1 & 146.74 & 23.65 & $97.86-193.38$ \\
\hline HR2 & 143.02 & 21.70 & $100.64-188.08$ \\
\hline HR3 & 136.20 & 20.39 & 105.18-191.65 \\
\hline HR4 & 133.68 & 18.48 & $99.82-179.83$ \\
\hline HR5 & 132.24 & 16.53 & $103.18-170.58$ \\
\hline RSAB & 4.00 & 1.26 & $1.22-7.08$ \\
\hline RSAO & 4.02 & 1.92 & $0-7.58$ \\
\hline RSA1 & 3.45 & 1.75 & $0.14-8.15$ \\
\hline RSA2 & 4.43 & 1.45 & $0-7.45$ \\
\hline RSA3 & 4.02 & 1.52 & $0.18-7.91$ \\
\hline RSA4 & 4.02 & 1.52 & $0.49-7.59$ \\
\hline RSA5 & 4.12 & 1.38 & $1.12-6.90$ \\
\hline
\end{tabular}

FLACC, Face, Legs, Activity, Cry, Consolability scale; HR, heart rate (beats per minute); RSA, respiratory sinus arrhythmia.

and each postneedle HR response significantly positively predicted the subsequent $\mathrm{HR}$ response (standardized Bs = 0.70-0.91).

\subsubsection{Predictive between-measure}

Higher HR preneedle (HRB) significantly predicted a lower behavioral pain response immediately after the vaccination (FLACC0) (standardized $B=-0.19, P=0.05$ ). As well, higher HR 90 seconds after the vaccination (HR3) significantly predicted a higher behavioral pain response 120 seconds after the vaccination (FLACC4) (standardized $B=0.23, P=0.01$ ). No other HR epoch significantly predicted behavioral pain scores across the vaccination period. Next, a higher behavioral pain response immediately after the vaccination (FLACCO) significantly predicted toddlers' HR 30 seconds after the vaccination (HR1) (standardized $\mathrm{B}=0.25, P<0.001$ ). None of the other 6 behavioral pain response epochs significantly predicted subsequent $\mathrm{HR}$ responses.

\subsubsection{Concurrent (residual) between-measure}

Heart rate and behavioral pain responses remained significantly positively related controlling for their predictors in the model at each epoch (residual $r s=0.42-0.58$ ); however, the concurrent residual relations were not significant immediately (residual $r=$ $0.12, P=0.19$ ) or 60 seconds (residual $r=0.23, P=0.09$ ) after the vaccination. 


\section{Table 5}

\section{Correlations among heart rate variables observed at 12 months.}

\begin{tabular}{|c|c|c|c|c|c|c|c|c|c|c|c|c|c|c|c|c|}
\hline & 1 & 2 & 3 & 4 & 5 & 6 & 7 & 8 & 9 & 10 & 11 & 12 & 13 & 14 & 15 & 16 \\
\hline 1. Time since last feeding & - & 0.11 & 0.02 & 0.01 & -0.07 & -0.15 & -0.10 & -0.86 & -0.13 & 0.09 & 0.08 & 0.11 & 0.07 & 0.05 & 0.05 & 0.00 \\
\hline 2. Time since last nap & & - & 0.02 & 0.00 & 0.02 & 0.07 & 0.02 & 0.06 & -0.04 & $0.23^{*}$ & 0.02 & $0.25^{\star}$ & 0.16 & 0.05 & 0.18 & 0.13 \\
\hline 3. HRB & & & - & $0.57^{*}$ & $0.49^{\star}$ & $0.53^{*}$ & $0.60^{*}$ & $0.65^{\star}$ & $0.60^{*}$ & $0.56^{*}$ & 0.03 & 0.11 & 0.14 & 0.14 & $0.25^{*}$ & 0.15 \\
\hline 4. HRO & & & & - & $0.72^{\star}$ & $0.61^{*}$ & $0.51^{*}$ & $0.47^{\star}$ & $0.42^{\star}$ & $0.40^{*}$ & 0.18 & 0.14 & $0.20^{*}$ & 0.12 & 0.15 & 0.14 \\
\hline 5. HR1 & & & & & - & $0.85^{\star}$ & $0.74^{*}$ & $0.65^{*}$ & $0.51^{*}$ & $0.39^{*}$ & $0.36^{*}$ & $0.45^{\star}$ & $0.42^{*}$ & $0.31^{*}$ & $0.35^{*}$ & $0.30^{*}$ \\
\hline 6. HR2 & & & & & & - & $0.88^{*}$ & $0.75^{\star}$ & $0.61^{*}$ & $0.35^{\star}$ & $0.30^{*}$ & $0.40^{*}$ & $0.46^{*}$ & $0.32^{*}$ & $0.35^{*}$ & $0.30^{*}$ \\
\hline 7. HR3 & & & & & & & - & $0.86^{\star}$ & $0.72^{*}$ & $0.27^{\star}$ & $0.25^{\star}$ & $0.31^{*}$ & $0.37^{\star}$ & $0.39^{*}$ & $0.45^{\star}$ & $0.32^{*}$ \\
\hline 8. HR4 & & & & & & & & - & $0.77^{\star}$ & $0.20^{*}$ & 0.09 & $0.22^{*}$ & $0.26^{*}$ & $0.34^{*}$ & $0.53^{*}$ & $0.34^{*}$ \\
\hline 9. HR5 & & & & & & & & & - & $0.23^{*}$ & 0.14 & 0.15 & $0.20^{*}$ & $0.22^{*}$ & $0.39^{*}$ & $0.38^{*}$ \\
\hline 10. FLACCB & & & & & & & & & & - & $0.29^{*}$ & $0.34^{*}$ & $0.23^{*}$ & $0.24^{*}$ & $0.27^{*}$ & $0.27^{\star}$ \\
\hline 11. FLACCO & & & & & & & & & & & - & $0.70^{*}$ & $0.55^{\star}$ & $0.38^{*}$ & $0.33^{*}$ & $0.35^{\star}$ \\
\hline 12. FLACC1 & & & & & & & & & & & & - & $0.76^{*}$ & $0.54^{*}$ & $0.44^{*}$ & $0.42^{*}$ \\
\hline 13. FLACC2 & & & & & & & & & & & & & - & $0.64^{*}$ & $0.49^{*}$ & $0.48^{*}$ \\
\hline 14. FLACC3 & & & & & & & & & & & & & & - & $0.74^{*}$ & $0.65^{\star}$ \\
\hline 15. FLACC4 & & & & & & & & & & & & & & & - & $0.73^{*}$ \\
\hline 16. FLACC5 & & & & & & & & & & & & & & & & - \\
\hline
\end{tabular}

${ }^{*}$ Correlation is significant at $<0.05$ level (2-tailed).

FLACC, Face, Legs, Activity, Cry, Consolability scale; HR, heart rate.

\subsubsection{Contextual factors}

At 12 months, baseline pain behaviors (FLACCB) significantly predicted subsequent pain behaviors immediately (FLACCO) after the vaccination (standardized $B=0.40, P<0.001$ ). Time since last nap (standardized $B=-0.06, P=0.44$ ) and last feeding (standardized $\mathrm{B}=0.05, P=0.46$ ) did not significantly predict pain behaviors (FLACCO) immediately after the vaccination. Baseline HR (HRB) significantly predicted HR immediately (HRO) after the vaccination (standardized $B=0.47, P<0.001$ ). Time since last nap (standardized $B=-0.00, P=0.98$ ) and last feeding (standardized $\mathrm{B}=-0.02, P=0.78$ ) did not significantly predict $\mathrm{HR}(\mathrm{HRO})$ immediately after the vaccination.

\section{Table 6}

\section{Correlations among respiratory sinus arrhythmia variables observed at 12 months.}

\begin{tabular}{|c|c|c|c|c|c|c|c|c|c|c|c|c|c|c|c|c|}
\hline & 1 & 2 & 3 & 4 & 5 & 6 & 7 & 8 & 9 & 10 & 11 & 12 & 13 & 14 & 15 & 16 \\
\hline 1. Time since last feeding & - & 0.11 & 0.02 & 0.01 & -0.07 & -0.15 & -0.10 & -0.09 & -0.13 & 0.09 & 0.08 & 0.11 & 0.07 & 0.05 & 0.05 & 0.000 \\
\hline 2. Time since last nap & & - & -0.14 & -0.06 & -0.00 & 0.01 & 0.08 & -0.07 & -0.02 & $0.23^{*}$ & 0.02 & $0.25^{*}$ & 0.16 & 0.05 & 0.18 & 0.13 \\
\hline 3. RSAB & & & - & $0.29^{*}$ & $0.23^{*}$ & $0.40^{*}$ & $0.43^{\star}$ & $0.42^{*}$ & $0.58^{\star}$ & $-0.42^{\star}$ & -0.16 & -0.14 & -0.05 & -0.04 & -0.10 & 0.03 \\
\hline 4. RSAO & & & & - & $0.29^{*}$ & $0.23^{*}$ & 0.16 & $0.28^{\star}$ & 0.16 & $-0.32^{\star}$ & -0.04 & 0.02 & 0.03 & 0.05 & 0.07 & 0.01 \\
\hline 5. RSA1 & & & & & - & $0.41^{*}$ & $0.43^{\star}$ & $0.31^{*}$ & $0.21^{*}$ & $-0.33^{*}$ & $-0.23^{\star}$ & $-0.33^{*}$ & $-0.24^{*}$ & -0.16 & -0.08 & -0.09 \\
\hline 6. RSA2 & & & & & & - & $0.57^{\star}$ & $0.44^{*}$ & $0.40^{*}$ & $-0.29^{\star}$ & -0.08 & -0.16 & -0.16 & 0.06 & 0.04 & 0.04 \\
\hline 7. RSA3 & & & & & & & - & $0.47^{\star}$ & $0.46^{*}$ & -0.17 & $-0.21^{*}$ & $-0.23^{*}$ & -0.12 & -0.07 & -0.06 & 0.00 \\
\hline 8. RSA4 & & & & & & & & - & $0.43^{*}$ & $-0.21^{\star}$ & 0.00 & -0.10 & -0.02 & 0.00 & -0.11 & 0.01 \\
\hline 9. RSA5 & & & & & & & & & - & -0.15 & -0.01 & 0.08 & 0.11 & 0.07 & 0.00 & 0.04 \\
\hline 10. FLACCB & & & & & & & & & & - & $0.29^{*}$ & $0.34^{*}$ & $0.23^{*}$ & $0.24^{*}$ & $0.27^{\star}$ & $0.27^{\star}$ \\
\hline 11. FLACCO & & & & & & & & & & & - & $0.70^{*}$ & $0.55^{*}$ & $0.38^{*}$ & $0.33^{*}$ & $0.35^{*}$ \\
\hline 12. FLACC1 & & & & & & & & & & & & - & $0.76^{\star}$ & $0.54^{*}$ & $0.44^{\star}$ & $0.42^{*}$ \\
\hline 13. FLACC2 & & & & & & & & & & & & & - & $0.64^{*}$ & $0.49^{*}$ & $0.48^{*}$ \\
\hline 14. FLACC3 & & & & & & & & & & & & & & - & $0.74^{\star}$ & $0.65^{\star}$ \\
\hline 15. FLACC4 & & & & & & & & & & & & & & & - & $0.73^{*}$ \\
\hline 16. FLACC5 & & & & & & & & & & & & & & & & - \\
\hline
\end{tabular}

* Correlation is significant at $<0.05$ level (2-tailed).

FLACC, Face, Legs, Activity, Cry, Consolability scale; RSA, respiratory sinus arrhythmia. 


\section{Table 7}

\section{Correlations among heart rate variables observed at 18 months.}

\begin{tabular}{|c|c|c|c|c|c|c|c|c|c|c|c|c|c|c|c|c|}
\hline & 1 & 2 & 3 & 4 & 5 & 6 & 7 & 8 & 9 & 10 & 11 & 12 & 13 & 14 & 15 & 16 \\
\hline 1. Time since last feeding & - & 0.11 & 0.09 & 0.06 & 0.14 & 0.13 & 0.06 & 0.06 & 0.08 & -0.05 & 0.05 & 0.10 & 0.09 & 0.07 & 0.08 & 0.02 \\
\hline 2. Time since last nap & & - & -0.22 & $-0.24^{*}$ & -0.11 & 0.01 & 0.07 & 0.02 & -0.02 & -0.11 & -0.13 & -0.07 & -0.09 & -0.08 & -0.10 & -0.10 \\
\hline 3. HRB & & & - & $0.75^{*}$ & $0.57^{\star}$ & $0.46^{\star}$ & $0.43^{*}$ & $0.42^{*}$ & $0.57^{\star}$ & $0.67^{\star}$ & 0.20 & 0.19 & 0.20 & $0.23^{*}$ & 0.18 & $0.31^{*}$ \\
\hline 4. HRO & & & & - & $0.82^{*}$ & $0.66^{\star}$ & $0.58^{*}$ & $0.48^{*}$ & $0.55^{\star}$ & $0.59^{*}$ & $0.55^{\star}$ & $0.41^{*}$ & $0.36^{*}$ & $0.32^{\star}$ & $0.34^{*}$ & $0.36^{*}$ \\
\hline 5. HR1 & & & & & - & $0.85^{\star}$ & $0.66^{*}$ & $0.54^{*}$ & $0.52^{*}$ & $0.56^{*}$ & $0.60^{\star}$ & $0.62^{*}$ & $0.56^{*}$ & $0.44^{\star}$ & $0.36^{\star}$ & $0.36^{*}$ \\
\hline 6. HR2 & & & & & & - & $0.83^{*}$ & $0.74^{*}$ & $0.65^{\star}$ & $0.42^{*}$ & $0.48^{*}$ & $0.58^{*}$ & $0.63^{\star}$ & $0.50^{*}$ & $0.40^{\star}$ & $0.39^{\star}$ \\
\hline 7. HR3 & & & & & & & - & $0.88^{*}$ & $0.79^{*}$ & $0.26^{*}$ & $0.30^{*}$ & $0.35^{*}$ & $0.46^{\star}$ & $0.49^{\star}$ & $0.41^{*}$ & $0.39^{*}$ \\
\hline 8. HR4 & & & & & & & & - & $0.87^{*}$ & 0.21 & $0.25^{\star}$ & $0.23^{*}$ & $0.36^{\star}$ & $0.43^{*}$ & $0.46^{*}$ & $0.39^{\star}$ \\
\hline 9. HR5 & & & & & & & & & - & $0.31^{*}$ & 0.17 & 0.16 & $0.30^{*}$ & $0.41^{*}$ & $0.47^{\star}$ & $0.50^{\star}$ \\
\hline 10. FLACCB & & & & & & & & & & - & $0.39^{*}$ & $0.40^{\star}$ & $0.40^{\star}$ & $0.42^{*}$ & $0.43^{\star}$ & $0.52^{\star}$ \\
\hline 11. FLACCO & & & & & & & & & & & - & $0.66^{*}$ & $0.59^{*}$ & $0.41^{*}$ & $0.41^{*}$ & $0.31^{*}$ \\
\hline 12. FLACC1 & & & & & & & & & & & & - & $0.74^{*}$ & $0.57^{*}$ & $0.51^{*}$ & $0.43^{\star}$ \\
\hline 13. FLACC2 & & & & & & & & & & & & & - & $0.81^{*}$ & $0.67^{\star}$ & $0.57^{\star}$ \\
\hline 14. FLACC3 & & & & & & & & & & & & & & - & $0.82^{*}$ & $0.72^{*}$ \\
\hline 15. FLACC4 & & & & & & & & & & & & & & & - & $0.85^{\star}$ \\
\hline 16. FLACC5 & & & & & & & & & & & & & & & & - \\
\hline
\end{tabular}

* Correlation is significant at $<0.05$ level (2-tailed).

FLACC, Face, Legs, Activity, Cry, Consolability scale; HR, heart rate.

\subsection{Relations between pain behaviors and respiratory sinus arrhythmia}

The autoregressive cross-lagged path model in Figure 2 fits the data adequately $(\mathrm{CFI}=0.88$; RMSEA $=0.08)$. Standardized estimates of significant paths are reported in Figure 2, and all [T10] standardized and unstandardized estimates are reported in Table 10.

\subsubsection{Predictive within-measure}

Across the vaccination period at 12 months, each postneedle behavioral response significantly positively predicted the subsequent behavioral response (standardized $\mathrm{Bs}=0.67-0.76$ ), and each postneedle RSA response significantly positively predicted the subsequent RSA response (standardized Bs = 0.30-0.57).

\section{Table 8}

\section{Correlations among respiratory sinus arrhythmia variables observed at 18 months.}

\begin{tabular}{|c|c|c|c|c|c|c|c|c|c|c|c|c|c|c|c|c|}
\hline & 1 & 2 & 3 & 4 & 5 & 6 & 7 & 8 & 9 & 10 & 11 & 12 & 13 & 14 & 15 & 16 \\
\hline 1. Time since last feeding & - & 0.11 & -0.03 & -0.07 & 0.02 & 0.02 & 0.07 & 0.15 & 0.10 & -0.05 & 0.05 & 0.10 & 0.09 & 0.07 & 0.08 & 0.02 \\
\hline 2. Time since last nap & & - & 0.10 & 0.11 & 0.08 & 0.02 & -0.09 & 0.02 & -0.04 & -0.11 & -0.13 & -0.07 & -0.09 & -0.08 & -0.10 & -0.10 \\
\hline 3. RSAB & & & - & $0.43^{\star}$ & $0.46^{\star}$ & $0.43^{*}$ & $0.44^{*}$ & $0.43^{*}$ & $0.59^{*}$ & $-0.32^{*}$ & -0.02 & -0.03 & -0.01 & -0.06 & -0.03 & -0.11 \\
\hline 4. RSAO & & & & - & $0.43^{*}$ & $0.38^{*}$ & $0.46^{*}$ & $0.43^{*}$ & $0.43^{*}$ & -0.19 & -0.12 & 0.00 & -0.04 & -0.08 & -0.13 & -0.15 \\
\hline 5. RSA1 & & & & & - & $0.56^{\star}$ & $0.43^{*}$ & $0.41^{*}$ & $0.33^{*}$ & $-0.27^{\star}$ & $-0.24^{*}$ & $-0.35^{\star}$ & $-0.30^{*}$ & -0.20 & -0.14 & -0.10 \\
\hline 6. RSA2 & & & & & & - & $0.69^{*}$ & $0.67^{\star}$ & $0.58^{\star}$ & -0.20 & $-0.24^{*}$ & $-0.27^{\star}$ & $-0.36^{*}$ & $-0.31^{\star}$ & $-0.23^{*}$ & $-0.22^{*}$ \\
\hline 7. RSA3 & & & & & & & - & $0.80^{*}$ & $0.72^{*}$ & 0.00 & -0.11 & -0.02 & -0.12 & -0.14 & -0.11 & -0.12 \\
\hline 8. RSA4 & & & & & & & & - & $0.70^{*}$ & -0.01 & -0.03 & 0.07 & 0.00 & -0.05 & -0.05 & -0.04 \\
\hline 9. RSA5 & & & & & & & & & - & -0.08 & 0.06 & 0.12 & 0.05 & -0.06 & -0.04 & -0.10 \\
\hline 10. FLACCB & & & & & & & & & & - & $0.39^{\star}$ & $0.40^{*}$ & $0.40^{*}$ & $0.42^{*}$ & $0.43^{*}$ & $0.52^{\star}$ \\
\hline 11. FLACCO & & & & & & & & & & & - & $0.66^{*}$ & $0.59^{*}$ & $0.41^{*}$ & $0.41^{*}$ & $0.31^{*}$ \\
\hline 12. FLACC1 & & & & & & & & & & & & - & $0.74^{*}$ & $0.57^{\star}$ & $0.51^{*}$ & $0.43^{*}$ \\
\hline 13. FLACC2 & & & & & & & & & & & & & - & $0.81^{*}$ & $0.67^{\star}$ & $0.57^{*}$ \\
\hline 14. FLACC3 & & & & & & & & & & & & & & - & $0.82^{\star}$ & $0.72^{\star}$ \\
\hline 15. FLACC4 & & & & & & & & & & & & & & & - & $0.85^{*}$ \\
\hline 16. FLACC5 & & & & & & & & & & & & & & & & - \\
\hline
\end{tabular}

* Correlation is significant at $<0.05$ level (2-tailed).

FLACC, Face, Legs, Activity, Cry, Consolability scale; RSA, respiratory sinus arrhythmia. 


\section{Table 9}

Estimates for autoregressive cross-lagged path model of relations between behavioural pain scores and heart rate during 12-month vaccination.

\begin{tabular}{|c|c|c|c|c|}
\hline & $\begin{array}{l}\text { Standardized } \\
\text { estimate }\end{array}$ & $\begin{array}{l}\text { Unstandardized } \\
\text { estimate }\end{array}$ & $z$ & $P$ \\
\hline \multicolumn{5}{|l|}{ FLACCO } \\
\hline FLACCB & 0.40 & 0.35 & 4.13 & $<0.001$ \\
\hline HRB & -0.19 & -0.02 & -1.95 & 0.05 \\
\hline $\begin{array}{l}\text { Time since last } \\
\text { nap }\end{array}$ & -0.06 & -0.00 & -0.77 & 0.44 \\
\hline $\begin{array}{l}\text { Time since last } \\
\text { fed }\end{array}$ & 0.05 & 0.00 & 0.74 & 0.46 \\
\hline \multicolumn{5}{|l|}{ HRO } \\
\hline HRB & 0.47 & 0.74 & 4.66 & $<0.001$ \\
\hline FLACCB & 0.15 & 1.75 & 1.65 & 0.10 \\
\hline $\begin{array}{l}\text { Time since last } \\
\text { nap }\end{array}$ & -0.00 & -0.00 & -0.03 & 0.98 \\
\hline $\begin{array}{l}\text { Time since last } \\
\text { fed }\end{array}$ & -0.02 & -0.01 & -0.28 & 0.78 \\
\hline \multicolumn{5}{|l|}{ FLACC1 } \\
\hline FLACCO & 0.69 & 1.12 & 10.25 & $<0.001$ \\
\hline HRO & 0.04 & 0.01 & 0.67 & 0.51 \\
\hline \multicolumn{5}{|l|}{ HR1 } \\
\hline HRO & 0.70 & 0.76 & 11.11 & $<0.001$ \\
\hline FLACCO & 0.25 & 3.80 & 5.21 & $<0.001$ \\
\hline \multicolumn{5}{|l|}{ FLACC2 } \\
\hline FLACC1 & 0.72 & 0.83 & 10.70 & $<0.001$ \\
\hline HR1 & 0.07 & 0.01 & 1.04 & 0.30 \\
\hline \multicolumn{5}{|l|}{ HR2 } \\
\hline HR1 & 0.85 & 0.77 & 18.18 & $<0.001$ \\
\hline FLACC1 & 0.01 & 0.11 & 0.28 & 0.78 \\
\hline \multicolumn{5}{|l|}{ FLACC3 } \\
\hline FLACC2 & 0.63 & 0.63 & 8.66 & $<0.001$ \\
\hline HR2 & 0.05 & 0.01 & 0.56 & 0.58 \\
\hline \multicolumn{5}{|l|}{ HR3 } \\
\hline HR2 & 0.91 & 0.82 & 19.70 & $<0.001$ \\
\hline FLACC2 & -0.05 & -0.35 & -1.28 & 0.20 \\
\hline \multicolumn{5}{|l|}{ FLACC4 } \\
\hline FLACC3 & 0.65 & 0.64 & 8.77 & $<0.001$ \\
\hline HR3 & 0.23 & 0.03 & 2.69 & 0.01 \\
\hline \multicolumn{5}{|l|}{ HR4 } \\
\hline HR3 & 0.88 & 0.0 .81 & 13.51 & $<0.001$ \\
\hline FLACC3 & -0.03 & -0.21 & -0.61 & 0.54 \\
\hline \multicolumn{5}{|l|}{ FLACC5 } \\
\hline FLACC4 & 0.71 & 0.72 & 6.87 & $<0.001$ \\
\hline HR4 & -0.04 & -0.01 & -0.38 & 0.70 \\
\hline \multicolumn{5}{|l|}{ HR5 } \\
\hline HR4 & 0.84 & 0.82 & 13.58 & $<0.001$ \\
\hline FLACC4 & -0.13 & -0.83 & -1.22 & 0.22 \\
\hline
\end{tabular}

FLACC, Face, Legs, Activity, Cry, Consolability scale; HR, heart rate.

\subsubsection{Predictive between-measure}

Higher RSA 60 seconds after the vaccination (RSA2) significantly predicted a higher behavioral pain response 90 seconds after the vaccination ( $F L A C C 3)(B=0.16, P=0.02)$. No other RSA epoch significantly predicted behavioral pain scores across the vaccination period. Next, a higher behavioral pain response preneedle (FLACCB) significantly predicted lower RSA immediately after the vaccination (RSAO) (standardized $B$ $=-0.22, P=0.03)$. As well, a higher behavioral pain response immediately after the vaccination (FLACCO) significantly
Table 10

Estimates for autoregressive cross-lagged path model of relations between behavioural pain scores and respiratory sinus arrhythmia during 12-month vaccination.

\begin{tabular}{|c|c|c|c|c|}
\hline & $\begin{array}{l}\text { Standardized } \\
\text { estimate }\end{array}$ & $\begin{array}{l}\text { Unstandardized } \\
\text { estimate }\end{array}$ & $\mathbf{z}$ & $P$ \\
\hline \multicolumn{5}{|l|}{ FLACCO } \\
\hline FLACCB & 0.27 & 0.23 & 3.82 & $<0.001$ \\
\hline RSAB & -0.06 & -0.08 & -0.72 & 0.47 \\
\hline $\begin{array}{l}\text { Time since last } \\
\text { nap }\end{array}$ & -0.05 & -0.00 & -0.62 & 0.54 \\
\hline $\begin{array}{l}\text { Time since last } \\
\text { fed }\end{array}$ & 0.04 & 0.00 & 0.74 & 0.46 \\
\hline \multicolumn{5}{|l|}{ RSAO } \\
\hline RSAB & 0.18 & 0.35 & 1.38 & 0.17 \\
\hline FLACCB & -0.22 & -0.26 & -2.12 & 0.03 \\
\hline $\begin{array}{l}\text { Time since last } \\
\text { nap }\end{array}$ & -0.01 & -0.00 & -0.06 & 0.95 \\
\hline $\begin{array}{l}\text { Time since last } \\
\text { fed }\end{array}$ & 0.04 & 0.00 & 0.44 & 0.66 \\
\hline \multicolumn{5}{|l|}{ FLACC1 } \\
\hline FLACCO & 0.70 & 1.12 & 10.61 & $<0.001$ \\
\hline RSAO & 0.05 & 0.05 & 0.77 & 0.44 \\
\hline \multicolumn{5}{|l|}{ RSA1 } \\
\hline RSAO & 0.30 & 0.21 & 3.31 & 0.001 \\
\hline FLACCO & -0.23 & -0.23 & -3.10 & 0.002 \\
\hline \multicolumn{5}{|l|}{ FLACC2 } \\
\hline FLACC1 & 0.76 & 0.87 & 13.61 & $<0.001$ \\
\hline RSA1 & 0.02 & 0.03 & 0.26 & 0.79 \\
\hline \multicolumn{5}{|l|}{ RSA2 } \\
\hline RSA1 & 0.39 & 0.33 & 4.00 & $<0.001$ \\
\hline FLACC1 & -0.03 & -0.01 & -0.33 & 0.74 \\
\hline \multicolumn{5}{|l|}{ FLACC3 } \\
\hline FLACC2 & 0.67 & 0.67 & 11.14 & $<0.001$ \\
\hline RSA2 & 0.16 & 0.35 & 2.37 & 0.02 \\
\hline \multicolumn{5}{|l|}{ RSA3 } \\
\hline RSA2 & 0.57 & 0.54 & 6.21 & $<0.001$ \\
\hline FLACC2 & -0.03 & -0.01 & -0.35 & 0.73 \\
\hline \multicolumn{5}{|l|}{ FLACC4 } \\
\hline FLACC3 & 0.74 & 0.72 & 13.04 & $<0.001$ \\
\hline RSA3 & -0.03 & -0.06 & -0.30 & 0.76 \\
\hline \multicolumn{5}{|l|}{ RSA4 } \\
\hline RSA3 & 0.47 & 0.43 & 5.01 & $<0.001$ \\
\hline FLACC3 & 0.05 & 0.02 & 0.61 & 0.54 \\
\hline \multicolumn{5}{|l|}{ FLACC5 } \\
\hline FLACC4 & 0.74 & 0.75 & 12.37 & $<0.001$ \\
\hline RSA4 & 0.12 & 0.29 & 1.51 & 0.13 \\
\hline \multicolumn{5}{|l|}{ RSA5 } \\
\hline RSA4 & 0.44 & 0.50 & 5.31 & $<0.001$ \\
\hline FLACC4 & 0.05 & 0.02 & 0.36 & 0.72 \\
\hline
\end{tabular}

FLACC, Face, Legs, Activity, Cry, Consolability scale; RSA, respiratory sinus arrhythmia.

predicted lower RSA 30 seconds after the vaccination (RSA1) (standardized $B=-0.23, P=0.002$ ). None of the other behavioral pain response epochs significantly predicted subsequent RSA responses.

\subsubsection{Concurrent (residual) between-measure}

Respiratory sinus arrhythmia and behavioral pain responses remained significantly negatively related controlling for their predictors in the model prevaccination, as well as 30- and 
120-seconds postvaccination (residual $r s=-0.25$ to -0.41 ). The concurrent residual relations were not significant immediately (residual $r=0.09, P=0.35$ ), 60 (residual $r=-0.06, P$ $=0.49$ ), 90 (residual $r=-0.18, P=0.09$ ), or 120 seconds (residual $r=-0.03, P=0.77$ ) after the vaccination.

\subsubsection{Contextual factors}

At 12 months, baseline pain behaviors significantly predicted subsequent pain behaviors immediately (FLACCO) after the vaccination (standardized $B=0.27, P<0.001$ ). Time since last nap (standardized $\mathrm{B}=-0.05, P=0.54$ ) and last feeding (standardized $\mathrm{B}=0.04, P=0.46$ ) did not significantly predict pain behaviors (FLACCO) immediately after the vaccination. Baseline RSA did not significantly predict RSA immediately (RSA0) after the vaccination (standardized $\mathrm{B}=0.47, P<$ 0.001). Time since last nap (standardized $B=-0.01, P=$ 0.95 ) and last feeding (standardized $B=0.04, P=0.66$ ) did not significantly predict $\mathrm{HR}(\mathrm{HRO})$ immediately after the vaccination.

\subsection{The relations between toddlers' behavioral and cardiac responses during 18-month vaccinations}

\subsubsection{Relations between pain behaviors and heart rate}

The autoregressive cross-lagged path model in Figure 3 fits the data adequately $(\mathrm{CFI}=0.96$; RMSEA $=0.07)$. Standardized estimates of significant paths are reported in Figure 3, and all [T11] standardized and unstandardized estimates are reported in Table 11.

\subsubsection{Predictive within-measure}

Across the vaccination period at 18 months, each postneedle behavioral response significantly positively predicted the subsequent behavioral response (standardized $\mathrm{Bs}=0.62$ 0.85 ) and each postneedle HR response significantly positively predicted the subsequent $\mathrm{HR}$ response (standardized Bs = 0.72-0.91).

\subsubsection{Predictive between-measure}

No HR epoch significantly predicted subsequent behavioral pain scores across the vaccination period. However, a higher behavioral pain response immediately after the vaccination (FLACCO) significantly predicted toddlers' HR 30 seconds after the vaccination $(H R 1)$ (standardized $B=0.20, P=0.001$ ). No other behavioral pain response epochs significantly predicted subsequent HR responses.

\subsubsection{Concurrent (residual) between-measure}

Heart rate and behavioral pain responses remained significantly positively related controlling for their predictors in the model at each epoch (residual $r s=0.37-0.66$ ).

\subsubsection{Contextual factors}

At 18 months, baseline pain behaviors (FLACCB) significantly predicted subsequent pain behaviors immediately (FLACCO) after the vaccination (standardized $B=0.45, P<0.001$ ). Time since last nap (standardized $\mathrm{B}=-0.10, P=0.34$ ) and last feeding (standardized $B=0.06, P=0.45$ ) did not significantly predict pain behaviors (FLACCO) immediately after the vaccination. Baseline HR (HRB) significantly AQ:6 predicted $\mathrm{HR}$ immediately $(\mathrm{HRO})$ after the vaccination
Table 11

Estimates for autoregressive cross-lagged path model of relations between behavioural pain scores and heart rate during 18-month vaccination.

\begin{tabular}{|c|c|c|c|c|}
\hline & $\begin{array}{l}\text { Standardized } \\
\text { estimate }\end{array}$ & $\begin{array}{l}\text { Unstandardized } \\
\text { estimate }\end{array}$ & $z$ & $P$ \\
\hline \multicolumn{5}{|l|}{ FLACCO } \\
\hline FLACCB & 0.45 & 0.35 & 4.11 & $<0.001$ \\
\hline HRB & -0.11 & -0.01 & -0.90 & 0.37 \\
\hline $\begin{array}{l}\text { Time since last } \\
\text { nap }\end{array}$ & -0.10 & -0.00 & -0.96 & 0.34 \\
\hline $\begin{array}{l}\text { Time since last } \\
\text { fed }\end{array}$ & 0.06 & 0.00 & 0.75 & 0.45 \\
\hline \multicolumn{5}{|l|}{ HRO } \\
\hline HRB & 0.65 & 0.85 & 7.13 & $<0.001$ \\
\hline FLACCB & 0.13 & 1.11 & 1.70 & 0.09 \\
\hline $\begin{array}{l}\text { Time since last } \\
\text { nap }\end{array}$ & -0.13 & -0.03 & -1.84 & 0.07 \\
\hline $\begin{array}{l}\text { Time since last } \\
\text { fed }\end{array}$ & -0.03 & -0.01 & -0.36 & 0.72 \\
\hline \multicolumn{5}{|l|}{ FLACC1 } \\
\hline FLACCO & 0.62 & 0.86 & 7.08 & $<0.001$ \\
\hline HRO & 0.07 & 0.01 & 0.76 & 0.45 \\
\hline \multicolumn{5}{|l|}{ HR1 } \\
\hline HRO & 0.72 & 0.75 & 10.18 & $<0.001$ \\
\hline FLACCO & 0.20 & 2.23 & 3.32 & 0.001 \\
\hline \multicolumn{5}{|l|}{ FLACC2 } \\
\hline FLACC1 & 0.66 & 0.66 & 8.27 & $<0.001$ \\
\hline HR1 & 0.13 & 0.02 & 1.46 & 0.14 \\
\hline \multicolumn{5}{|l|}{ HR2 } \\
\hline HR1 & 0.82 & 0.75 & 8.43 & $<0.001$ \\
\hline FLACC1 & 0.04 & 0.31 & 0.44 & 0.66 \\
\hline \multicolumn{5}{|l|}{ FLACC3 } \\
\hline FLACC2 & 0.82 & 0.82 & 12.18 & $<0.001$ \\
\hline HR2 & -0.00 & -0.01 & -0.07 & 0.95 \\
\hline \multicolumn{5}{|l|}{ HR3 } \\
\hline HR2 & 0.86 & 0.84 & 11.22 & $<0.001$ \\
\hline FLACC2 & -0.05 & -0.34 & -0.59 & 0.55 \\
\hline \multicolumn{5}{|l|}{ FLACC4 } \\
\hline FLACC3 & 0.80 & 0.79 & 11.34 & $<0.001$ \\
\hline HR3 & 0.01 & 0.01 & 0.48 & 0.63 \\
\hline \multicolumn{5}{|l|}{ HR4 } \\
\hline HR3 & 0.91 & 0.81 & 12.77 & $<0.001$ \\
\hline FLACC3 & -0.04 & -0.29 & -0.70 & 0.48 \\
\hline \multicolumn{5}{|l|}{ FLACC5 } \\
\hline FLACC4 & 0.85 & 0.91 & 17.34 & $<0.001$ \\
\hline HR4 & -0.01 & -0.00 & -0.21 & 0.83 \\
\hline \multicolumn{5}{|l|}{ HR5 } \\
\hline HR4 & 0.84 & 0.76 & 12.02 & $<0.001$ \\
\hline FLACC4 & 0.05 & 0.33 & 0.86 & 0.39 \\
\hline
\end{tabular}

(standardized $\mathrm{B}=0.65, P<0.001$ ). Time since last nap (standardized $B=-0.13, P=0.07$ ) and last feeding (standardized $\mathrm{B}=-0.03, P=0.72$ ) did not significantly predict HR (HRO) immediately after the vaccination.

\subsection{Relations between pain behaviors and respiratory sinus arrhythmia}

The autoregressive cross-lagged path model in Figure 4 fits the data adequately $(\mathrm{CFI}=0.93$; RMSEA $=0.08)$. Standardized estimates of 
[T12] significant paths are reported in Figure 3, and all standardized and unstandardized estimates are reported in Table 12.

\subsubsection{Predictive within-measure}

Across the vaccination period at 18 months, each postneedle behavioral response significantly positively predicted the subsequent behavioral response (standardized Bs $=0.67$ 0.84) and each postneedle RSA response significantly

Table 12

Estimates for autoregressive cross-lagged path model of relations between behavioural pain scores and respiratory sinus arrhythmia during 18-month vaccination.

\begin{tabular}{|c|c|c|c|c|}
\hline & $\begin{array}{l}\text { Standardized } \\
\text { estimate }\end{array}$ & $\begin{array}{l}\text { Unstandardized } \\
\text { estimate }\end{array}$ & $z$ & $P$ \\
\hline \multicolumn{5}{|l|}{ FLACCO } \\
\hline FLACCB & 0.42 & 0.32 & 5.60 & $<0.001$ \\
\hline RSAB & 0.12 & 0.20 & 1.11 & 0.27 \\
\hline $\begin{array}{l}\text { Time since last } \\
\text { nap }\end{array}$ & -0.11 & -0.00 & -1.03 & 0.30 \\
\hline $\begin{array}{l}\text { Time since last } \\
\text { fed }\end{array}$ & 0.08 & 0.00 & 1.03 & 0.30 \\
\hline \multicolumn{5}{|l|}{ RSAO } \\
\hline RSAB & 0.39 & 0.60 & 4.51 & $<0.001$ \\
\hline FLACCB & -0.06 & -0.04 & -0.49 & 0.63 \\
\hline $\begin{array}{l}\text { Time since last } \\
\text { nap }\end{array}$ & -0.06 & 0.00 & 0.68 & 0.50 \\
\hline $\begin{array}{l}\text { Time since last } \\
\text { fed }\end{array}$ & -0.05 & -0.00 & -0.51 & 0.61 \\
\hline \multicolumn{5}{|l|}{ FLACC1 } \\
\hline FLACCO & 0.67 & 0.93 & 10.42 & $<0.001$ \\
\hline RSAO & 0.09 & 0.14 & 1.23 & 0.22 \\
\hline \multicolumn{5}{|l|}{ RSA1 } \\
\hline RSAO & 0.40 & 0.43 & 4.50 & $<0.001$ \\
\hline FLACCO & -0.16 & -0.19 & -2.38 & 0.02 \\
\hline \multicolumn{5}{|l|}{ FLACC2 } \\
\hline FLACC1 & 0.74 & 0.73 & 12.36 & $<0.001$ \\
\hline RSA1 & -0.06 & -0.02 & -0.20 & 0.84 \\
\hline \multicolumn{5}{|l|}{ RSA2 } \\
\hline RSA1 & 0.44 & 0.54 & 4.79 & $<0.001$ \\
\hline FLACC1 & -0.03 & -0.06 & -0.60 & 0.55 \\
\hline \multicolumn{5}{|l|}{ FLACC3 } \\
\hline FLACC2 & 0.80 & 0.79 & 15.44 & $<0.001$ \\
\hline RSA2 & -0.02 & -0.05 & -0.44 & 0.66 \\
\hline \multicolumn{5}{|l|}{ RSA3 } \\
\hline RSA2 & 0.72 & 0.76 & 8.16 & $<0.001$ \\
\hline FLACC2 & 0.14 & 0.08 & 1.57 & 0.12 \\
\hline \multicolumn{5}{|l|}{ FLACC4 } \\
\hline FLACC3 & 0.82 & 0.81 & 15.64 & $<0.001$ \\
\hline RSA3 & 0.00 & 0.01 & 0.05 & 0.96 \\
\hline \multicolumn{5}{|l|}{ RSA4 } \\
\hline RSA3 & 0.81 & 0.81 & 11.45 & $<0.001$ \\
\hline FLACC3 & 0.08 & 0.04 & 1.13 & 0.26 \\
\hline \multicolumn{5}{|l|}{ FLACC5 } \\
\hline FLACC4 & 0.84 & 0.90 & 20.87 & $<0.001$ \\
\hline RSA4 & -0.00 & -0.00 & -0.01 & 1.0 \\
\hline \multicolumn{5}{|l|}{ RSA5 } \\
\hline RSA4 & 0.70 & 0.63 & 9.30 & $<0.001$ \\
\hline FLACC4 & 0.02 & 0.01 & 0.23 & 0.82 \\
\hline
\end{tabular}

positively predicted the subsequent RSA response (standardized $\mathrm{Bs}=0.40-0.81$ ).

\subsubsection{Predictive between-measure}

No RSA epoch significantly predicted subsequent behavioral pain scores across the vaccination period. A higher behavioral pain response immediately after the vaccination (FLACCO) significantly predicted lower RSA 30 seconds after the vaccination (RSA1) (standardized $\mathrm{B}=-0.16, P=0.02$ ). None of the other behavioral pain response epochs significantly predicted subsequent RSA responses.

\subsubsection{Concurrent (residual) between-measure}

Respiratory sinus arrhythmia and behavioral pain responses remained significantly negatively related controlling for their predictors in the model prevaccination and 60 seconds postvaccination (residual $r s=-0.31$ to -0.37 ). The concurrent residual relations were not significant immediately (residual $r=$ $-0.07, P=0.38$ ), 60 (residual $r=-0.26, P=0.06$ ), 90 (residual $r=-0.10, P=0.39$ ), 120 (residual $r=-0.04, P=0.69$ ), or 150 seconds (residual $r=-0.15, P=0.18$ ) after the vaccination.

\subsubsection{Contextual factors}

At 18 months, baseline pain behaviors significantly predicted subsequent pain behaviors immediately (FLACCO) after the vaccination (standardized $B=0.42, P<0.001$ ). Time since last nap (standardized $B=-0.11, P=0.30$ ) and last feeding (standardized $\mathrm{B}=0.08, P=0.30$ ) did not significantly predict pain behaviors (FLACCO) immediately after the vaccination. Baseline RSA did not significantly predict RSA immediately (RSA0) after the vaccination (standardized $B=0.39, P<0.001$ ). Time since last nap (standardized $B=-0.06, P=0.50$ ) and last feeding (standardized $\mathrm{B}=-0.05, P=0.61$ ) did not significantly predict $\mathrm{HR}(\mathrm{HRO})$ immediately after the vaccination.

\section{Discussion}

To the best of our knowledge, this is the first longitudinal study of typically developing toddlers (ie, 12 and 18 months) to examine the convergence of commonly used behavioral and cardiac indicators (ie, HR, RSA) of acute pain-related distress. This study is novel in that the analyses examined predictive within-measure, predictive between-measure, and concurrent (residual) betweenmeasure relations among behavioral and cardiac indicators of acute pain-related distress at 12 and 18 months. In addition, contextual factors were investigated to determine whether baseline responses (ie, baseline behavioral pain scores, HR, and RSA) or time since last feeding or nap predict behavior or physiology postneedle. The following discussion focuses on developmental trends based on changes in the strength of relation within- or between-measures from 12 to 18 months. Differences in the relation based on cardiac indicator (ie, HR, $\mathrm{RSA}$ ) and timing of measurement (ie, pain reactivity vs regulation) are also discussed. In the following discussion, FLACCO, HRO, and RSAO reflect the peak pain-related distress response that occurs immediately after the needle (reactivity), whereas subsequent FLACC, HR, and RSA epochs (ie, 1-5) capture the process of pain-related distress regulation from the needle. This section ends with limitations of our research and implications for future research and clinical practice. 


\subsection{Within-measure relations}

Within-measure relations across indicators (ie, behavior, $\mathrm{HR}$, and RSA) were positive, with each postneedle behavioral or cardiac response positively predicting the subsequent behavioral or cardiac response. These findings confirm past research suggesting that FLACC has high stability within the acute pain context in toddlerhood, ${ }^{32}$ and cardiac indicators are stable within conditions. ${ }^{15}$ Regarding developmental trends inferred by changes in the strength of relations, there were moderate to strong withinmeasure relations for behavioral and $\mathrm{HR}$ responses across ages. However, within-measure relations for RSA were stronger at 18 months than at 12 months of age. Previous research ${ }^{42}$ has found age-related changes in RSA and noted that this reflects increased autonomic complexity across development. Indeed, weaker within-measure relations for RSA were found compared to behavioral pain scores and HR across ages, likely reflecting that RSA captures additional biopsychosocial aspects of the toddler's pain experience. ${ }^{42}$ In addition, the strongest within-measure autocorrelations for RSA were found within the regulatory epochs (ie, RSA1-RSA5), which is consistent with theories suggesting that RSA is more reflective of parasympathetic vs sympathetic influence. $^{4}$

\subsection{Predictive between-measure relations}

Across ages and cardiac indicators, behavioral pain responses immediately postneedle positively predicted HR and negatively predicted RSA 30 seconds postneedle. These results suggest that expressed pain behaviors may have a regulating or dysregulating impact on toddler physiology in the initial reactivity period of the vaccination, which affects the entire regulatory phase through other within-measure and concurrent relations. This predictive relation between behavioral pain response and RSA represents vagal influence being withdrawn due to increased sympathetic (ie, behavioral pain response) activation. ${ }^{6}$ In addition to these consistent findings across 12 and 18 months, there were significant pathways at 12 months of age. Specifically, higher baseline HR predicted lower behavioral pain responses immediately postneedle, whereas higher behavioral pain responses at baseline predicted lower RSA immediately postneedle. These divergent relations (ie, HR predicting behavior and behavior predicting RSA) within the baseline and reactivity epochs are consistent with past research on toddler distress regulation, where one stress response compensates for another. $^{30,47,55}$ As well, HR and RSA responses 30 seconds postneedle positively predicted behavioral pain responses 60 seconds postneedle. These results suggest that toddler physiology may predict subsequent behavior, but only within the regulatory phase postneedle.

\subsection{Concurrent (residual) between-measure relations}

Overall, there were concurrent associations between behavioral pain responses and each of HR and RSA at 12 and 18 months, over and above their predictive autoregressive and cross-lagged effects. As expected, behavioral and HR responses were positively related, and behavioral and RSA responses were negatively related. Regarding developmental differences, behavioral pain responses and $\mathrm{HR}$ were consistently related across the prevaccination and postvaccination periods at 18 months, but not at 12 months. Heart rate and behavioral pain responses were not significantly concurrently related immediately or 60 seconds after the vaccination at 12 months, over and above the contextual baseline factors. As such, it is possible that contextual baseline factors and previous behavioral pain responses and physiology more strongly predict certain postvaccination pain-related distress responses than other indicators measured concurrently. Alternatively, behavioral pain responses and HR may reflect unique aspects of the nociceptive response at 12 months of age. Indeed, Roué et al. ${ }^{45}$ found that some typically developing neonates presented with acute responses measured by physiological indicators, whereas others presented with prolonged stressful responses characterized by expressed pain behaviors.

Comparing the concurrent relation between behavioral pain responses and each of $\mathrm{HR}$ and $\mathrm{RSA}, \mathrm{HR}$ had stronger and more consistent relations with behavior than RSA. These differences in magnitude of the relation between behavioral and cardiac painrelated distress indicators are consistent with studies investigating pain responses in younger preterm infants ${ }^{29}$ and in nonpain contexts. 2,7,8,19,21,26,43 The strongest relations between behavioral pain responses and HR were within the reactivity phase, whereas the strongest associations between behavioral pain responses and RSA were within the regulation phases. These results confirm classic theories suggesting that the sympathetic nervous system is most associated with distress, ${ }^{14}$ whereas RSA may be more related to regulatory strategies that are aligned with the parasympathetic nervous system. ${ }^{25}$

\subsection{Contextual factors}

Baseline responses consistently predicted future pain scores and physiology within the postneedle period at both 12 and 18 months, whereas time since last feeding and time since last nap were not significantly related to behavioral pain scores and physiology at either age. Regarding developmental trends, baseline RSA only emerged as a significant predictor of future RSA scores within the postvaccination period at 18 months. The emergence of baseline RSA as a significant predictor of future RSA scores at 18 months may reflect the many regulatory abilities and skills that emerge in toddlerhood. ${ }^{11}$ Overall, these results are in line with the Law of Initial Value ${ }^{5}$ but extend the theory to baseline behavioral pain scores in addition to physiology.

\subsection{Limitations}

Despite having sample size comparable to other studies of neonatal pain assessment, ${ }^{3,12,27,37,45,50}$ generalizability of the current results is affected by the high education level of our participants. As well, our study included healthy toddlers born full-term who underwent a standardized acutely painful procedure, which limits generalizability to nonhealthy neonates or premature infants who must undergo multiple acute painful or stressful procedures.

\subsection{Conclusions: clinical and research implications}

In this study, normative data were provided regarding how commonly used behavioral and cardiac pain-related distress indicators are related within the acute pain context in toddlerhood. Our findings suggest that compared to RSA, HR is more strongly related and closely linked to behavioral pain indicators in toddlerhood. The shared yet unique variance between HR and pain behaviors suggests that these indicators would be complementary measures of pain in toddlers, and confirms practice in neonatology where multimodal approaches to pain in those nonverbal children is the evidence-based bedside approach. ${ }^{17}$ Indeed, reliable differences in behavioral pain-related distress have been found in 12 -month-old infants, ${ }^{41}$ with 
environmental factors (ie, attachment relationship with primary caregiver) leading some typically developing infants to exhibit less behavioral distress following vaccinations. ${ }^{18}$ Based on the unique variance shared between HR and pain behaviors found in the current study, multimodal approaches that incorporate both cardiac and behavioral indicators are hypothesized to more accurately capture infant pain-related distress, especially for infants with dampened behavioral pain responses. However, there are several challenges related to utility of current infant pain assessment tools (ie, physiological, cortical, behavioral indicators). ${ }^{39}$ There is a lack of specificity to pain for physiological measures, ${ }^{39}$ behavioral indicators (ie, facial actions, body movements, and cry) are not reliably associated with parental judgments of infant pain, ${ }^{40}$ and interventions to alleviate pain have been shown to reduce behavioral pain scores without altering nociceptive brain and spinal cord activity. ${ }^{51}$ Indeed, there is consensus among basic and clinical scientists that cortical, physiological, and behavioral measures of pain do not consistently converge. ${ }^{39}$ Therefore, this research supports that use of $\mathrm{HR}$ in conjunction with expressed behavioral pain and other physiological and cortical indicators (eg, oxygen saturation, electroencephalography, skin conductance, and cortisol) to properly encapsulate the nociceptive response in toddlers. Although positive within-measure relations were found among behavioral and cardiac indicators across ages, behavioral and cardiac indicators of pain-related distress are not consistently concurrently related to each other after accounting for the withinand between-measure predictors as well as contextual factors (ie, baseline responses). Given the inconsistent concurrent relations between indicators after accounting for predictive associations, pain scores may be misestimated if pain indicators are only used within the initial reactivity phase (eg, 30 seconds postneedle) without accounting for these contextual factors (ie, baseline responses). As mentioned previously, these inconsistent concurrent findings need to be also contextualized by the reciprocal relation between behavioral and cardiac indicators across the 12- and 18-month vaccinations. Specifically, across ages, behavioral pain responses immediately postneedle significantly predicted HR and RSA 30 seconds postneedle. It is important to consider the impact of toddlers' initial behavioral response on their physiological and behavioral regulation from pain-related distress. Covariates included in the models may also account for weaker concurrent residual relations because baseline behavioral pain scores and cardiac indicators significantly predicted subsequent pain scores and physiology within the postvaccination period. Pain assessment tools should measure behavioral and physiological responses at baseline as well as across the initial reactivity and regulatory phases to provide a more holistic understanding of the toddler's pain experience because both indicators predict future pain scores.

\section{Conflict of interest statement}

The authors have no conflicts of interest to declare.

\section{Acknowledgments}

This research was funded by awards to R.R. Pillai Riddell from the Natural Sciences and Engineering Research Council of Canada, the Ontario Ministry of Research and Innovation, and the Canadian Foundation for Innovation, as well as Doctoral Training Awards to J.A. Waxman and M.G. DiLorenzo from the Canadian Institutes of Health Research, Ontario Graduate Scholarship, and Meighen Wright Graduate Scholarship in Maternal-Child Health.
J.A. Waxman and M.G. DiLorenzo are trainee members of Pain In Child Health $(\mathrm{PICH})$, a strategic research training initiative of the Canadian Institutes of Health Research. Funders were not involved in the design or conduct of this study.

\section{Article history:}

Received 18 September 2019

Received in revised form 25 January 2020

Accepted 14 February 2020

Available online $X X X X$

\section{References}

[1] Anand KJ, Craig KD. New perspectives on the definition of pain. PAIN 1996;67:3-6.

[2] Baker E, Baibazarova E, Ktistaki G, Shelton KH, van Goozen SHM. Development of fear and guilt in young children: stability over time and relations with psychopathology. Dev Psychopathol 2012;24:833-45.

[3] Bartocci M, Bergqvist LL, Lagercrantz H, Anand KJ. Pain activates cortical areas in the preterm newborn brain. PAIN 2006;122:109-17.

[4] Berntson GG, Quigley KS, Lozano D. Cardiovascular psychophysiology. In: Cacioppo JT, Tassinary LG, Berntson GG, editors. Handbook of psychophysiology. New York: Cambridge University Press, 2007. pp. 182-210.

[5] Berntson GG, Uchino BN, Cacioppo JT. Origins of baseline variance and the law of initial values. Psychophysiology 1994;31:204-10.

[6] Bornstein MH, Suess PE. Child and mother cardiac vagal tone: continuity, stability, and concordance across the first 5 years. Dev Psychol 2000;36: $54-65$.

[7] Brooker RJ, Buss KA. Dynamic measures of RSA predict distress and regulation in toddlers. Dev Psychobiol 2010;52:372-82.

[8] Buss KA, Goldsmith HH, Davidson RJ. Cardiac reactivity is associated with changes in negative emotion in 24-month-olds. Dev Psychobiol 2005;46:118-32.

[9] Campbell L, Pillai Riddell R, Greenberg S, Garfield H. A cross-sectional examination of the relationships between caregiver proximal soothing and infant pain over the first year of life. PAIN 2013;154:813-23.

[10] Carbajal R, Paupe A, Hoenn E, Lenclen R, Olivier-Martin M. APN: evaluation behavioral scale of acute pain in newborn infants. Arch Pediatr 1997;4:623-8.

[11] Craig KD, Whitfield MF, Grunau RV, Linton J, Hadjistavropoulos HD. Pain in the preterm neonate: behavioral and physiological indices. PAIN 1993; 52:287-99.

[12] Eriksson M, Storm H, Fremming A, Schollin J. Skin conductance compared to a combined behavioral and physiological pain measure in newborn infants. Acta Paediatr 2008;97:27-30.

[13] Fietd T. The effeets of mothers' physieat and motional unavailability on

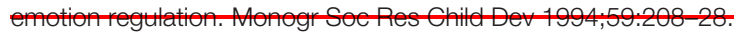

[14] Fowles D. Psychophysiology and psychopathology: a motivational approach. Psychophysiology 1988;25:373-91.

[15] Fracasso MP, Porges SW, Lamb ME, Rosenberg AA. Cardiac activity in infancy: reliability and stability of individual differences. Infant Behav Dev 1994;17:277-84.

[16] Gibbins S, Stevens B, McGrath PJ, Yamada J, Beyene J, Breau L, Camfield C, Finley A, Franck L, Johnston C, Howlett A, McKeever P, O'Brien K, Ohlsson A. Comparison of pain responses in infants of different gestational ages. Neonatology 2008;93:10-18.

[17] Gibbins S, Stevens BJ, Yamada J, Dionne K, Campbell-Yeo M, Lee G, Caddell K, Johnston C, Taddio A. Validation of the premature infant pain profile-revised (PIPP-R). Early Hum Dev 2014;90:189-93.

[18] Horton R, Pillai Riddell R, Flora D, Moran G, Pederson D. Distress regulation in infancy: attachment and temperament in the context of acute pain. J Dev Behav Pediatr 2015;36:35-44.

[19] Hay DF, van Goozen SHM, Mundy L, Phillips R, Roberts S, Meeuwsen M, Goodyer I, Perra O. If you go down to the woods today: infants' distress during a teddy bear's picnic in relation to peer relations and later emotional problems. Infancy 2017;22:552-70.

[20] Holsti L, Grunau RE. Initial validation of the behavioral indicators of infant pain (BIIP). PAIN 2007;132:264-72.

[21] Holzman JB, Bridgett DJ. Heart rate variability indices as bio-markers of top-down self-regulatory mechanisms: a meta-analytic review. Neurosci Biobehav Rev 2017;74:233-55.

[22] Huffman LC, Bryan YE, Carmen R, Pedersen FA, Doussard-Roosevelt JA, Porges SW. Infant temperament and cardiac vagal tone: assessments at twelve weeks of age. Child Development 1998;69:624-35. 
[23] H Hum elP, Lawtor-Klean P, Weiss MG. Validity and reliability of the $\mathrm{N}-$ PASS

[24] Jones L, Fabrizi L, Laudiano-Dray M, Whitehead K, Meek J, Verriotis M, Fitzgerald M. Nociceptive cortical activity is dissociated from nociceptive behavior in newborn human infants under stress. Curr Biol 2017;27:1-6.

[25] Jonsdottir RB, Kristjansdottir G. The sensitivity of the premature infant pain profile-PIPP to measure pain in hospitalized neonates. J Eval Clin Pract 2005;11:598-605.

[26] Kahle S, Miller JG, Helm JL, Hastings PD. Linking autonomic physiology and emotion regulation in preschoolers: the role of reactivity and recovery. Dev Psychbio 2018;60:775-88.

[27] Karpe J, Misiołek A, Daszkiewicz A, Misiołek H. Objective assessment of pain-related stress in mechanically ventilated newborns based on skin conductance fluctuations. Anaesthesiol Intensive Ther 2013;45:134-7.

[28] Kessler RC, Greenberg DF. Linear panel analysis. New York: Academic Press, 1981.

[29] Lucas-Thompson R, Townsend EL, Gunnar MR, Georgieff MK, Guiang SF, Ciffuentes RF, Lussky RC, Davis EP. Developmental changes in the responses of preterm infants to a painful stressor. Infant Behav Dev 2008; 31:614-23.

[30] McEwen BS. Physiology and neurobiology of stress and adaptation: central role of the brain. Physiol Rev 2007;87:873-904.

[31] McGrath PF, Unruh AM. Measurement and assessment of paediatric pain. In: Wall PD, Melzack R, editors. Textbook of pain. New York: Churchill Livingstone, 1994. pp. 303-12.

[32] Merkel S, Voepel-Lewis T, Malviya S. Pain assessment in infants and young children: the FLACC scale: a behavioral tool to measure pain in young children. Am J Nurs 2002;102:55-8.

[33] Merkel S, Voepel-Lewis T, Shayevitz JR, Malviya S. The FLACC: a behavioral scale for scoring postoperative pain in young children. Pediatr Nurs 1997;23:293-7.

[34] Morison SJ, Grunau RE, Oberlander TF, Whitfield MF. Relations between behavioral and cardiac autonomic reactivity to acute pain in preterm neonates. Clin J Pain 2001;17:350-8.

[35] Novak V, Saul JP, Eckberg DL. Task force report on heartrate variability. Circulation 1997;96:1056-7.

[36] Oberlander T, Saul JP. Methodological considerations for the use of heart rate variability as a measure of pain reactivity in vulnerable infants. Clin Perinatology 2002;29:427-43.

[37] Pereira-da-Silva L, Virella D, Monteiro I, Gomes S, Rodrigues P, Serelha $\mathrm{M}$, Storm $H$. Skin conductance indices discriminate nociceptive responses to acute stimuli from different heel prick procedures in infants. J Matern Fetal Neonatal Med 2012;25:796-801.

[38] Peters JW, Koot HM, Grunau RE, de Boer J, van Druenen MJ, Tibboel D, Duivenvoorden HJ. Neonatal facial coding system for assessing postoperative pain in infants: item reduction is valid and feasible. Clin $\mathrm{J}$ Pain 2003;19:353-63.

[39] Pillai Riddell R, Fitzgerald M, Slater R, Stevens B, Johnston C, CampbellYeo M. Using only behaviors to assess infant pain: a painful compromise? PAIN 2016;157:1579-80.

[40] Pillai Riddell R, Flora DB, Stevens S, Greenberg S, Garfield H. The role of infant pain beahavior in predicting parent pain ratings. Pain Res Manag 2014;19:e124-32.

[41] Pillai Riddell R, Flora DB, Stevens SA, Stevens B, Cohen LL, Greenberg S, Garfield $\mathrm{H}$. Variability in infant acute pain responding meaningfully obscured by averaging pain responses. PAIN 2013;154:714-21.
[42] Porges SW. The polyvagal perspective. Biol Psychol 2007;74:116-43.

[43] Provost MA, Gouin-Decarie T. Heart rate reactivity of 9- and 12-months old infants showing specific emotions in natural setting. Int J Behav Dev 1979;2:109-20.

[44] Ranger M, Celeste Johnston C, Rennick JE, Limperopoulos C, Heldt $\mathrm{T}$, du Plessis AJ. A multidimensional approach to pain assessment in critically ill infants during a painful procedure. Clin J Pain 2013;29: 613-20.

[45] Roué JM, Rioualen S, Gendras J, Misery L, Gouillou M, Sizun J. Multimodal pain assessment: are near-infrared spectroscopy, skin conductance, salivary cortisol, physiologic parameters, and Neonatal Facial Coding System interrelated during venepuncture in healthy, term neonates? J Pain Res 2018;11:2257-67.

[46] Santucci AK, Silk JS, Shaw DS, Gentzler A, Fox NA, Kovacs M. Vagal tone and temperament as predictors of emotion regulation strategies in young children. Dev Psychobio 2008;50:205-16.

[47] Sapolsky RM, Romero LM, Munck AU. How do glucocorticoids influence stress responses? Integrating permissive, suppressive, stimulatory, and preparative actions. Endocr Rev 2000;21:55-89.

[48] Schneider J, Duerden EG, Guo T, Ng K, Hagmann P, Bickle Graz M, Grunau RE, Chakravarty MM, Hüppi PS, Truttmann AC, Miller SP. Procedural pain and oral glucose in preterm neonates: brain development and sex-specific effects. PAIN 2018;159:515-25.

[49] Schwaller F, Fitzgerald M. The consequences of pain in early life: injuryinduced plasticity in developing pain pathways. Eur J Neurosci 2014;39: 344-52.

[50] Slater R, Cantarella A, Franck L, Meek J, Fitzgerald M. How well do clinical pain assessment tools reflect pain in infants? Plos Med 2008;5:e129.

[51] Slater R, Cornelissen L, Fabrizi L, Patten D, Yoxen J, Worley A, Boyd S, Meek J, Fitzgerald M. Oral sucrose as an analgesic drug for procedural pain in newborn infants: a randomised controlled trial. Lancet 2010;376: 1225-32.

[52] Taddio A, Appleton M, Bortolussi R, Chambers C, Dubey V, Halperin S, Hanrahan A, Ipp M, Lockett D, MacDonald N, Midmer D, Mousmanis P, Palda V, Pielak K, Riddell RP, Rieder M, Scott J, Shah V. Reducing the pain of childhood vaccination: an evidence-based clinical practice guideline. CMAJ 2010;182:E843-55.

[53] Task Force of the European Society of Cardiology and the North American Society of Pacing and Electrophysiology. Heart rate variability: standards of measurement, physiological interpretation and clinical use. Task force of the European society of cardiology and the north American society of pacing and electrophysiology. Circulation 1996;93:1043-65.

[54] Valeri BO, Ranger CMY, Chai IL, Cepeda IL, Synnes A, Linhares MB, Grunau RE. Neonatal invasive procedures predict pain intensity at school age in children born very preterm. Clin J Pain 2016;32:1086-93.

[55] van Stegeren AH, Wolf OT, Kindt M. Salivary alpha amylase and cortisol responses to different stress tasks: impact of sex. Int J Psychophysiol 2008;69:33-40.

[56] von Baeyer CL. Children's self-reports of pain intensity: scale selection, limitations and interpretation. Pain Res Manag 2006;11:157-62.

[57] Waxman J, DiLorenzo M, Pillai Riddell R. Convergence of behavioral and cardiovascular indicators of distress in toddlerhood: A systematic review and narrative synthesis. 2019; Manuscript submitted for publication.

[58] Waxman JA, Pillai Riddell RR, Pinhasov A, Schmidt LA, Tablon P. Development of cardiovascular indexes of acute pain responding in infants: a systematic review. Pain Res Manag 2016;16:1-16. 
000 An examination of the reciprocal and concurrent relations between behavioral and cardiac indicators of acute pain in toddlerhood

Concurrent and cross-lagged relations between behavioral and cardiac indicators of pain were inconsistent in toddlerhood. Potentially different dimensions of pain may be reflected.

Jordana A. Waxman, Miranda G. DiLorenzo, Rebecca R. Pillai Riddell, David B. Flora, Louis A. Schmidt, Hartley Garfield, Dan Flanders, Eitan Weinberg, Deena Savlov 